\title{
Finite-momentum condensate of magnetic excitons in a bilayer quantum Hall system
}

\author{
R. L. Doretto, ${ }^{1}$ C. Morais Smith, ${ }^{2}$ and A. O. Caldeira ${ }^{3}$ \\ ${ }^{1}$ Instituto de Física Teórica, Universidade Estadual Paulista, 01140-070 São Paulo, SP, Brazil \\ ${ }^{2}$ Institute for Theoretical Physics, Utrecht University, 3584 CE Utrecht, The Netherlands \\ ${ }^{3}$ Instituto de Física Gleb Wataghin, Universidade Estadual de Campinas, 13083-970 Campinas, SP, Brazil
}

(Received 8 March 2012; revised manuscript received 2 July 2012; published 26 July 2012)

\begin{abstract}
We study the bilayer quantum Hall system at total filling factor $v_{T}=1$ within a bosonization formalism which allows us to approximately treat the magnetic exciton as a boson. We show that in the region where the distance between the two layers is comparable to the magnetic length, the ground state of the system can be seen as a finite-momentum condensate of magnetic excitons provided that the excitation spectrum is gapped. We analyze the stability of such a phase within the Bogoliubov approximation first assuming that only one momentum $\mathbf{Q}$ is macroscopically occupied and later we consider the same situation for two modes $\pm \mathbf{Q}$. We find strong evidences that a first-order quantum phase transition at small interlayer separation takes place from a zero-momentum condensate phase, which corresponds to Halperin 111 state, to a finite-momentum condensate of magnetic excitons.
\end{abstract}

DOI: 10.1103/PhysRevB.86.035326

PACS number(s): 73.21.Ac, 73.43.Cd, 73.43.Lp, 73.43.Nq

\section{INTRODUCTION}

A bilayer quantum Hall system (QHS) consists of two two-dimensional electron gases (layers) separated by a small distance $d$ under a uniform magnetic field $\mathbf{B}$ perpendicular to the layers. Among the several possible configurations, we consider the one where each layer has filling factor $v=n \phi_{0} / B=1 / 2$, such that the total filling factor $v_{T}=$ $1 / 2+1 / 2=1$. Here, $n$ is the electronic density of each layer and $\phi_{0}=h c / e$ the magnetic flux quantum. ${ }^{1,2}$

The system is characterized by two parameters: the ratios $d / \ell$ and $\Delta_{\mathrm{SAS}} / E_{c}$. Here, $\ell=\sqrt{\hbar c / e B}$ is the magnetic length, the characteristic length scale of QHSs, $\Delta_{\text {SAS }}$ is the electron interlayer tunneling energy, and $E_{c}=e^{2} / \epsilon \ell$ is the characteristic Coulomb energy with $\epsilon$ being the dielectric constant of the host semiconductor. Although $\Delta_{\mathrm{SAS}}$ and the distance $d$ are fixed for a given sample, the ratio $d / \ell$ can be modified by changing the magnetic field $\mathbf{B}$ and then adjusting the electronic density in each layer in such a way that the configuration $v_{T}=1 / 2+1 / 2=1$ is restored. Interestingly, a series of measurements ${ }^{3-5}$ have shown that for $d<d_{c} \approx 1.8 \ell$, the bilayer QHS behaves as a single-layer QHS at $v=1$, while for $d>d_{c}$, as two independent two-dimensional electron gases at $v=1 / 2$. In spite of the fact that the experimental data indicate a continuous transition between these two situations, the so-called incompressible-compressible quantum phase transition, from the theoretical point of view it is not clear whether the system undergoes a second-order quantum phase transition $^{6}$ or a first-order one smeared out by disorder. ${ }^{7,8}$

The ground state of the bilayer QHS at $v_{T}=1$ is well understood in two limiting cases: for small $d / \ell$, it can be described by the (incompressible) Halperin 111 wave function, ${ }^{9}$ while in the very large $d / \ell$ region, by two independent (compressible) composite fermion Fermi liquids. ${ }^{10,11}$ Interestingly, the Halperin 111 state can be seen as a BoseEinstein condensate (BEC) of magnetic excitons, where the electron and the hole are in different layers. ${ }^{12}$ This analogy motivated us to employ the bosonization scheme ${ }^{13}$ to study the bilayer QHS at $v_{T}=1$. Our main finding in this first study ${ }^{14}$ was that a zero-momentum BEC of magnetic excitons is stable only for $d \leqslant 0.4 \ell$ (zero interlayer tunneling case). Such a result is in quite good agreement with the exact diagonalization calculations on finite-size systems, which show that the overlap between the exact ground state and the 111 state is close to unit only for $d \lesssim 0.5 \ell \ell^{15,16}$

Although much theoretical work ${ }^{6,8,15-22}$ has been devoted to the intermediate region $d \sim \ell$, so far there is no consensus about the nature of the ground state. For instance, a (pseudospin) density wave, ${ }^{8}$ mixed Fermi-Bose trial wave functions, ${ }^{15}$ and a (pseudospin) spiral state ${ }^{21}$ have been proposed as possible candidates. A proper description of the ground state in the intermediate $d / \ell$ region is important since it will help us to determine the nature of the incompressiblecompressible phase transition.

In this paper, we revisit the bilayer QHS within the bosonization formalism ${ }^{13,14}$ focusing on the intermediate $d / \ell$ region. We propose that within this bosonic scheme, the ground state of the system can be seen as a finite-momentum BEC of magnetic excitons. We show that this is indeed a possible phase of the effective boson model that we have derived in Ref. 14, provided that the (neutral) quasiparticle excitation spectrum is gapped. Our results also indicate that the instability of the zero-momentum BEC of magnetic excitons at $d=0.4 \ell$ reported in Ref. 14 indeed corresponds to a first-order quantum phase transition from a zero-momentum BEC of magnetic excitons to a finite-momentum one.

Our paper is organized as follows: In Sec. II, we introduce an interacting fermion model to describe the bilayer QHS, summarize the bosonization method, ${ }^{13}$ and recall the main steps to derive the effective boson model from the original fermionic one. We also comment on the motivation for considering a finite-momentum BEC of magnetic excitons as the ground state of the bilayer. Section III is devoted to the analysis of the effective boson model within the Bogoliubov approximation assuming that the ground state is given by a finite-momentum BEC of magnetic excitons where the momentum $\mathbf{Q}=Q \hat{x}$ is macroscopically occupied. The ground-state energy and the (neutral) quasiparticle excitation spectrum are calculated. Here, evidences that a first-order quantum phase transition takes place at small $d / \ell$ are found. In Sec. IV, we perform 
(a)

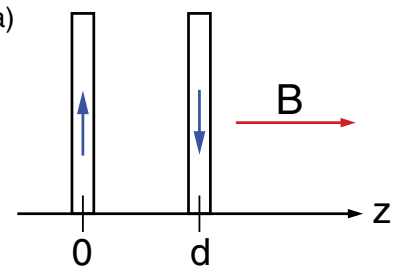

(b)

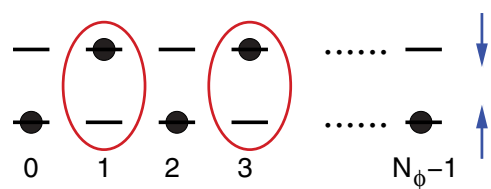

(c)

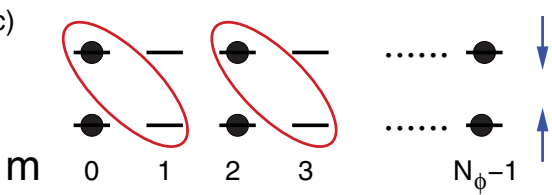

FIG. 1. (Color online) Schematic representations: (a) Bilayer QHS. B is the magnetic field and $d$ the distance between the two layers. (b) Zero-momentum BEC of magnetic excitons and (c) finite-momentum BEC of magnetic excitons with $|\ell \mathbf{Q}|=1$. Only the lowest Landau levels corresponding to the $\uparrow$ and $\downarrow$ layers are shown. $m$ is the guiding center quantum number which labels the degeneracy of each Landau level.

a similar analysis but now considering that two modes, $\pm \mathbf{Q}$ with $\mathbf{Q}=Q \hat{x} \neq 0$, are macroscopically occupied. We show that $\left|\ell \mathbf{Q}_{0}\right|$, the magnitude of the momentum associated with the lowest-energy configuration, increases with $d / \ell$. Some additional features of a BEC of magnetic excitons are shown in Sec. V. In Sec. VI, we compare our results with previous ones and comment on their consequences for the bilayer QHS at $v_{T}=1$. A short summary with the main results closes the paper. The fact that density fluctuations can account for the definition of boson operators for the bilayer QHS, comparison with alternative bosonic schemes used to describe the bilayer, and some details of the calculations can be found in the Appendixes.

\section{MODEL}

Let us consider a two-layer system composed of $N$ electrons moving in the $(x, y, z=0)$ plane and $N$ in the $(x, y, z=d)$ plane under an external magnetic field $\mathbf{B}=B \hat{z}$ [Fig. 1(a)] at zero temperature. We introduce a pseudospin index $\alpha=\uparrow$, $\downarrow$ in order to label each layer. We also assume that the B field is strong enough such that the electrons are fully spin polarized (frozen electronic spin degree of freedom) and that the Hilbert space of each layer is restricted to the corresponding lowest Landau level. The configuration $v_{T}=v_{\uparrow}+v_{\downarrow}=1 / 2+1 / 2=1$ is realized by setting the degeneracy of each Landau level $N_{\Phi}=2 N$.

The Hamiltonian of the system has only two terms (since all electrons are restricted to the lowest Landau level, the kinetic energy is a constant and can be neglected):

$$
H=H_{T}+H_{I} \text {. }
$$

Here, $H_{T}$ describes the electron tunneling between the two layers

$$
H_{T}=-\frac{1}{2} \Delta_{\mathrm{SAS}} \sum_{m} c_{m \uparrow}^{\dagger} c_{m \downarrow}+\text { H.c., }
$$

and $H_{I}$ is the Coulomb interaction term (we set the system area $\mathcal{A}=1$ )

$$
H_{I}=\frac{1}{2} \sum_{\mathbf{k} \neq 0} \sum_{\alpha \beta=\uparrow, \downarrow} v_{\alpha \beta}(k) \rho_{\alpha}(\mathbf{k}) \rho_{\beta}(-\mathbf{k})
$$

with $k=|\mathbf{k}| . \Delta_{\text {SAS }}$ is the electron interlayer tunneling energy, $c_{m \alpha}^{\dagger}$ creates an electron with guiding center $m$ in the lowest Landau level of the $\alpha$ layer [Fig. 1(b)], and $\rho_{\alpha}(\mathbf{k})$ is the Fourier transform of the $\alpha$-electron density operator projected into the lowest Landau level, i.e., ${ }^{13}$

$$
\rho_{\alpha}(\mathbf{k})=e^{-(\ell k)^{2} / 4} \sum_{m, m^{\prime}} G_{m, m^{\prime}}(\ell \mathbf{k}) c_{m \alpha}^{\dagger} c_{m^{\prime} \alpha} .
$$

The function $G_{m, m^{\prime}}(x)$ is defined in the Appendix A of Ref. 13. Finally,

$$
\begin{aligned}
& v_{\uparrow \uparrow}(k)=v_{\downarrow \downarrow}(k)=v_{A}(k)=\frac{2 \pi e^{2}}{\epsilon k} e^{-(\ell k)^{2} / 2}, \\
& v_{\uparrow \downarrow}(k)=v_{\downarrow \uparrow}(k)=v_{E}(k)=\frac{2 \pi e^{2}}{\epsilon k} e^{-(\ell k)^{2} / 2} e^{-k d}
\end{aligned}
$$

are, respectively, the Fourier transforms of the intralayer $v_{A}(r)=e^{2} / \epsilon r$ and interlayer $v_{E}(r)=e^{2} / \epsilon \sqrt{r^{2}+d^{2}}$ electronelectron interaction potentials with $r=|\mathbf{r}|$.

On can show that Eq. (2) can be written in terms of the $x$ component of the pseudospin density operator, i.e.,

$$
H_{T}=-\Delta_{\text {SAS }} S_{x}(\mathbf{k}=0),
$$

while Eq. (3), in terms of the total electron density operator $\rho(\mathbf{k})=\rho_{\uparrow}(\mathbf{k})+\rho_{\downarrow}(\mathbf{k})$ and the $z$ component of the pseudospin density operator $S_{z}(\mathbf{k})=\left[\rho_{\uparrow}(\mathbf{k})-\rho_{\downarrow}(\mathbf{k})\right] / 2$, namely,

$H_{I}=\frac{1}{2} \sum_{\mathbf{k} \neq 0} v_{0}(k) \rho(\mathbf{k}) \rho(-\mathbf{k})+2 \sum_{\mathbf{k} \neq 0} v_{z}(k) S_{z}(\mathbf{k}) S_{z}(-\mathbf{k})$,

with

$v_{0 / z}(k)=\frac{1}{2}\left[v_{A}(k) \pm v_{E}(k)\right]=\frac{\pi e^{2}}{\epsilon k} e^{-(\ell k)^{2} / 2}\left(1 \pm e^{-k d}\right)$.

In the following, we focus on the zero tunneling case, i.e., we set $\Delta_{\mathrm{SAS}}=0$ which yields $H=H_{I}$.

\section{A. Bosonization formalism}

We study the interacting fermion model (7) within the bosonization formalism ${ }^{13}$ that was recently developed by two of us among others. Although such a scheme was originally proposed for the single-layer QHS at $v=1$, it is possible to show that it also holds for the bilayer QHS at $\nu_{T}=1$ (see Appendix A). We now briefly summarize the bosonization method and refer the reader to Ref. 13 for more details. In Appendix B, we briefly comment on some alternative bosonic descriptions employed to study the bilayer QHS.

Let us consider the single-layer QHS at $v=1$. We restrict the Hilbert space to the lowest Landau level and explicitly take into account the electronic spin. The ground state of the system, the so-called quantum Hall ferromagnet $|\mathrm{FM}\rangle$, is illustrated in 
(a)

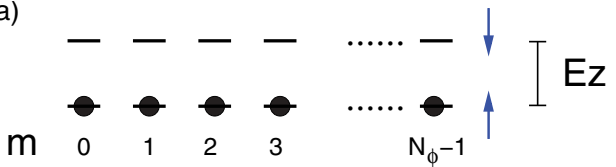

(b)

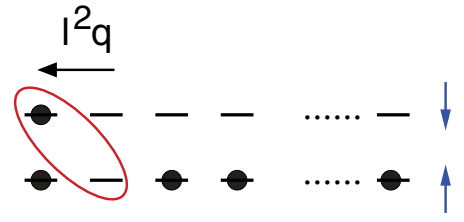

(c)

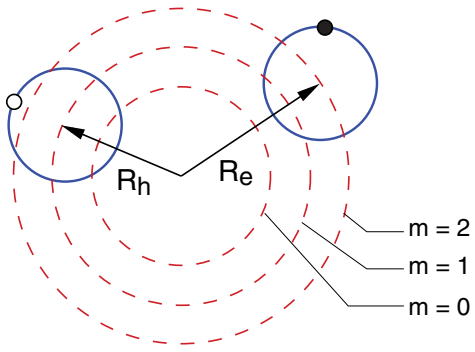

FIG. 2. (Color online) Schematic representations: (a) Quantum Hall ferromagnet $|\mathrm{FM}\rangle$, the ground state of the single-layer QHS at $v=1$, and (b) an electron-hole pair excitation (magnetic exciton) with momentum $|\ell \mathbf{q}|=1$ above $|\mathrm{FM}\rangle$. Only the spin-up and spin-down lowest Landau levels are shown. $E_{z}$ is the Zeeman energy and $m$ is the guiding center quantum number. (c) Semiclassical representation of an electron-hole pair in the symmetric gauge. Particles move along cyclotron orbits (solid blue circles), the center of which are at one of the possible guiding centers (dashed red circles). The vectors $\mathbf{R}_{e}$ and $\mathbf{R}_{h}$ correspond to the guiding center position of the electron (solid circle) and the hole (open circle), respectively.

Fig. 2(a). It is possible to show that the neutral elementary excitations above this state, electron-hole pairs also known as magnetic excitons [Fig. 2(b)], can be approximately treated as bosons. More precisely, we can define the following bosonic operators:

$$
\begin{aligned}
& b_{\mathbf{q}}=N_{\Phi}^{-1 / 2} e^{-(\ell q)^{2} / 4} \sum_{m, m^{\prime}} G_{m, m^{\prime}}(-\ell \mathbf{q}) c_{m \uparrow}^{\dagger} c_{m^{\prime} \downarrow}, \\
& b_{\mathbf{q}}^{\dagger}=N_{\Phi}^{-1 / 2} e^{-(\ell q)^{2} / 4} \sum_{m, m^{\prime}} G_{m, m^{\prime}}(\ell \mathbf{q}) c_{m \downarrow}^{\dagger} c_{m^{\prime} \uparrow},
\end{aligned}
$$

where $c_{m \sigma}^{\dagger}\left(c_{m \sigma}\right)$ is a creation (annihilation) operator for an electron in the lowest Landau level with guiding center $m$ and spin $\sigma$. The boson operators (9) obey the canonical commutation relations $\left[b_{\mathbf{q}}^{\dagger}, b_{\mathbf{k}}^{\dagger}\right]=\left[b_{\mathbf{q}}, b_{\mathbf{k}}\right]=0$ and $\left[b_{\mathbf{q}}, b_{\mathbf{k}}^{\dagger}\right]=\delta_{\mathbf{q}, \mathbf{k}}$ once some conditions are fulfilled. The state $b_{\mathbf{q}}^{\dagger}|\mathrm{FM}\rangle$ corresponds to a magnetic exciton with momentum q [Fig. 2(b)]. Within this framework, the electron density operator and the $z$ component of the spin density operator read as

$$
\begin{aligned}
& \rho(\mathbf{k})=\delta_{k, 0} N_{\Phi}+2 i \sum_{\mathbf{q}} \sin (\mathbf{k} \wedge \mathbf{q} / 2) b_{\mathbf{k}+\mathbf{q}}^{\dagger} b_{\mathbf{q}}, \\
& S_{z}(\mathbf{k})=\frac{1}{2} \delta_{k, 0} N_{\Phi}-\sum_{\mathbf{q}} \cos (\mathbf{k} \wedge \mathbf{q} / 2) b_{\mathbf{k}+\mathbf{q}}^{\dagger} b_{\mathbf{q}}
\end{aligned}
$$

with $\mathbf{k} \wedge \mathbf{q} \equiv \ell^{2} \hat{z} \cdot(\mathbf{k} \times \mathbf{q})$.
It is easy to see that, in principle, the bosonization scheme outlined above can be employed to study the bilayer QHS at $v_{T}=1$, once the pseudospin $\alpha$ is identified with the electronic spin quantum number $\sigma$ of the single-layer QHS at $v=1$ [compare Figs. 1(b) and 2(a) and recall that we consider that the electrons are completely spin polarized in the bilayer QHS]. Since the bosons $b$ are defined with respect to a reference state, the quantum Hall ferromagnet $|\mathrm{FM}\rangle$, the bilayer QHS at $v_{T}=1 / 2+1 / 2=1$ corresponds to a system with $N_{\Phi} / 2$ bosons, as illustrated in Fig. 1(b).

\section{B. Effective boson model}

Let us now follow the lines of Ref. 13 and map the original interacting fermion model (7) into an effective interacting boson model. Substituting Eqs. (10) and (11) into Eq. (7) and normal ordering the result, we arrive at

$$
H_{B}=\sum_{\mathbf{q}} \omega_{\mathbf{q}} b_{\mathbf{q}}^{\dagger} b_{\mathbf{q}}+\sum_{\mathbf{k} \neq 0, \mathbf{p}, \mathbf{q}} v_{\mathbf{k}}(\mathbf{p}, \mathbf{q}) b_{\mathbf{k}+\mathbf{p}}^{\dagger} b_{\mathbf{q}-\mathbf{k}}^{\dagger} b_{\mathbf{q}} b_{\mathbf{p}} .
$$

Here, ${ }^{23}$

$$
\omega_{\mathbf{q}}=\frac{e^{2}}{\epsilon \ell}\left[\sqrt{\frac{\pi}{2}}-\ell \int_{0}^{\infty} d k e^{-k d} e^{-(k \ell)^{2}} J_{0}\left(k q \ell^{2}\right)\right]
$$

is the dispersion relation of the free bosons (see Fig. 3), with $J_{0}(x)$ denoting the Bessel function of the first kind and

$$
\begin{aligned}
v_{\mathbf{k}}(\mathbf{p}, \mathbf{q})= & 2 v_{0}(k) \sin (\mathbf{k} \wedge \mathbf{p} / 2) \sin (\mathbf{k} \wedge \mathbf{q} / 2) \\
& +2 v_{z}(k) \cos (\mathbf{k} \wedge \mathbf{p} / 2) \cos (\mathbf{k} \wedge \mathbf{q} / 2)
\end{aligned}
$$

is the boson-boson interaction potential. In the following, instead of $H_{B}$, we consider

$$
K=H_{B}-\mu \hat{N},
$$

which explicitly includes the chemical potential $\mu$. Here, $\hat{N}=$ $\sum_{\mathbf{q}} b_{\mathbf{q}}^{\dagger} b_{\mathbf{q}}$ is the number operator for bosons.

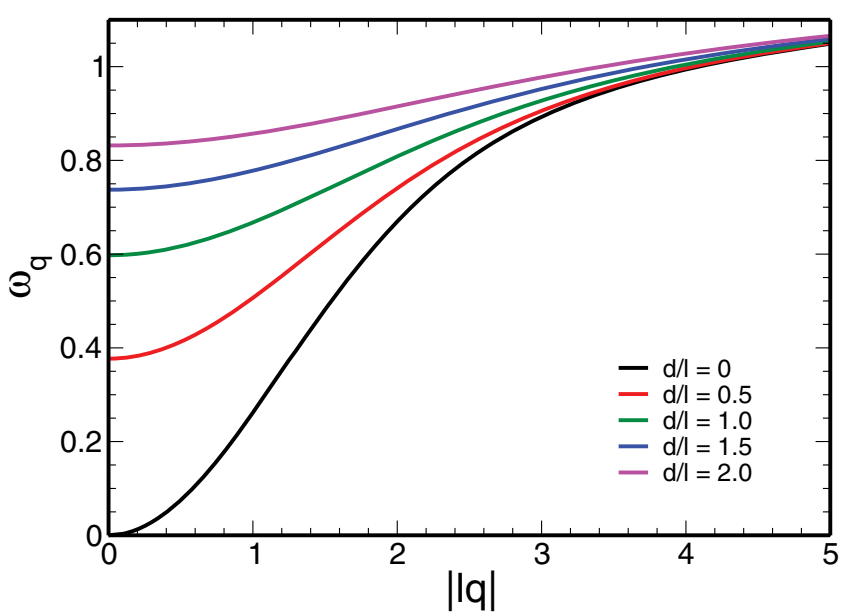

FIG. 3. (Color online) Dispersion relation of the free bosons (in units of $e^{2} / \epsilon \ell$ ) [Eq. (13)] for $d / \ell=0,0.5,1,1.5$, and 2 (from bottom to top at $\ell q=0$ ). 


\section{Finite-momentum BEC of magnetic excitons}

In Ref. 14, we analyzed the interacting boson model (12) assuming that the bosons $b$ condense in their lowest-energy state, the $\mathbf{q}=0$ mode, and showed that such a state is stable only for $d \leqslant 0.4 \ell$. The good agreement between our results and exact diagonalization calculations (see the Introduction section) tells us that not only is this zero-momentum BEC a good approximation for Halperin 111 state, but also that the bosonic formalism ${ }^{13}$ is indeed quite appropriate to describe the bilayer QHS at $v_{T}=1$. Therefore, it might be possible to describe the decrease of the correlations between the two layers as $d / \ell$ increases, i.e., the intermediate $d / \ell$ region, using solely the bosonic degrees of freedom. In this case, what should be the form of the ground state in terms of the bosons $b$ for $d \sim \ell$ ?

In order to construct the new ground state, we should recall some properties of the magnetic excitons. As mentioned above, the state $b_{\mathbf{q}}^{\dagger}|\mathrm{FM}\rangle$ corresponds to a magnetic exciton with momentum $\mathbf{q}$, which is nothing but a suitable linear combination of electron-hole pairs above the $|F M\rangle$ state [see Eq. (9)]. The momentum $\mathbf{q}$ is canonically conjugate to the vector $\mathbf{R}_{0}=\left(\mathbf{R}_{e}+\mathbf{R}_{h}\right) / 2,{ }^{13}$ where the vectors $\mathbf{R}_{e}$ and $\mathbf{R}_{h}$ denote, respectively, the position of the guiding centers of the electron and the hole as illustrated in Fig. 2(c). Interestingly, it is also possible to show that [see Eq. (2.16) in Ref. 24]

$$
\left\langle\mathrm{FM}\left|b_{\mathbf{q}}\left(\mathbf{R}_{e}-\mathbf{R}_{h}\right) b_{\mathbf{q}}^{\dagger}\right| \mathrm{FM}\right\rangle=\ell^{2} \mathbf{q} \times \hat{z},
$$

i.e., the (relative) distance between the guiding centers of the electron and the hole which constitute the magnetic exciton is $\propto q$. Note that this is an unusual relation between momentum and distance. Therefore, a boson $b$ with $\mathbf{q}=0$ can be seen as an electron-hole pair both localized in the same guiding center, while for a boson $b$ with $\mathbf{q} \neq 0$, the electron and the hole are in different guiding centers.

A zero-momentum BEC of magnetic excitons is then characterized by a large number of (interlayer) electron-hole pairs where each electron is very close (in the guiding center sense) to its partner hole as depicted in Fig. 1(b). Since this is the smallest distance between the electron and the hole, such a feature indicates that the two layers are highly correlated, corroborating the relation between the zero-momentum BEC and the 111 state. Therefore, in order to decrease the coupling between the two layers, we should, in principle, consider a state constituted of a large number of electron-hole pairs where now each electron is a little bit displaced from its partner hole. This situation is nothing but a finite-momentum BEC, where the bosons macroscopically occupy a finite $\mathbf{Q}$ mode, for instance, the one with $\ell Q=|\ell \mathbf{Q}|=1$ [Fig. 1 (c)]. Given such a relation between the momentum $\mathbf{Q}$ and interlayer coupling, we also expect that the larger $\ell Q$, the lower the correlation between the two layers.

These are the key points which motivated us to propose a finite-momentum BEC of magnetic excitons as a possible ground state for the bilayer QHS in the intermediate $d / \ell$ region. In the next two sections, we study the stability of this state at two different levels of approximation.

As a final remark, we should note that although finitemomentum BECs have been recently discussed in the context of ultracold Bose gases (see, for instance, Refs. 25 and 26), our motivation to consider such a phase is mainly due to the properties of the magnetic exciton as explained above.

\section{ONE-MODE APPROXIMATION}

In this section, we analyze the effective interacting boson model (12) within the so-called Bogoliubov approximation ${ }^{27}$ assuming that the ground state is given by a finite-momentum BEC with the $\mathbf{Q}=Q \hat{x}$ mode macroscopically occupied. We hereafter refer to this procedure as the one-mode approximation. Although the $\mathbf{Q}$ mode is not the lowest-energy single-particle boson state (see Fig. 3), we show that such a BEC is indeed a stable solution for certain values of $d / \ell$ provided that the excitation spectrum is gapped. Here, the boson-boson interaction potential (14) plays an important role in the stability of this phase. In the following, we consider $0.1 \leqslant \ell Q \leqslant 2$ and $0.1 \ell \leqslant d \leqslant 4 \ell$.

Before continuing, some words about the approximation scheme are here in order: since the single-particle boson energy (13) has cylindrical symmetry $\omega_{\mathbf{q}}=\omega_{q}$, there is no reason to expect that the bosons will condense in only one particular momentum $\mathbf{Q}=Q \hat{x}$. In principle, the bosons $b$ could even condense in more than one mode $\mathbf{q}$ as long as $q=Q$. However, such an approximation is the simplest one which allows us to verify whether a finite-momentum BEC of magnetic excitons is indeed a stable phase via quite accurate and well-controlled calculations. This is the idea of the procedure adopted in this section and in the next one. Later, in Sec. VI, we will discuss which of the features found here could be displayed by the bilayer QHS and also how the results derived from these two initial considerations could guide us to propose a more elaborated approximation scheme to study such a finite-momentum BEC.

We start by replacing $b_{\mathbf{Q}}^{\dagger}=b_{\mathbf{Q}}=\left\langle b_{\mathbf{Q}}^{\dagger}\right\rangle=\left\langle b_{\mathbf{Q}}\right\rangle \rightarrow \sqrt{N_{0}}$ in Eq. (15), where $N_{0}$ is the (macroscopic) number of bosons in the $\mathbf{Q}$ mode. Keeping only terms with two bosonic operators, one finds after some algebra that

$$
\begin{aligned}
K= & K_{0}+\frac{1}{2} \sum_{\mathbf{q} \neq 0}\left[\epsilon_{\mathbf{q}}^{+} b_{\mathbf{Q}+\mathbf{q}}^{\dagger} b_{\mathbf{Q}+\mathbf{q}}+\epsilon_{\mathbf{q}}^{-} b_{\mathbf{Q}-\mathbf{q}} b_{\mathbf{Q}-\mathbf{q}}^{\dagger}\right. \\
& \left.+\lambda_{\mathbf{q}}\left(b_{\mathbf{Q}+\mathbf{q}}^{\dagger} b_{\mathbf{Q}-\mathbf{q}}^{\dagger}+b_{\mathbf{Q}-\mathbf{q}} b_{\mathbf{Q}+\mathbf{q}}\right)\right],
\end{aligned}
$$

where

$$
\begin{aligned}
K_{0} & =\left(\omega_{\mathbf{Q}}-\mu\right) N_{0}-\frac{1}{2} \sum_{\mathbf{q} \neq 0} \epsilon_{\mathbf{q}}^{-}, \\
\epsilon_{\mathbf{q}}^{ \pm} & =\omega_{\mathbf{Q} \pm \mathbf{q}}-\mu+\lambda_{\mathbf{q}}, \\
\lambda_{\mathbf{q}} & =2 N_{0} v_{\mathbf{q}}(\mathbf{Q}, \mathbf{Q}) .
\end{aligned}
$$

The quadratic Hamiltonian (17) can be diagonalized with the aid of the canonical Bogoliubov transformation

$$
\begin{aligned}
& b_{\mathbf{Q}+\mathbf{q}}^{\dagger}=u_{\mathbf{q}} a_{\mathbf{Q}+\mathbf{q}}^{\dagger}-v_{\mathbf{q}} a_{\mathbf{Q}-\mathbf{q}}, \\
& b_{\mathbf{Q}-\mathbf{q}}^{\dagger}=u_{\mathbf{q}} a_{\mathbf{Q}-\mathbf{q}}^{\dagger}-v_{\mathbf{q}} a_{\mathbf{Q}+\mathbf{q}},
\end{aligned}
$$


(a)

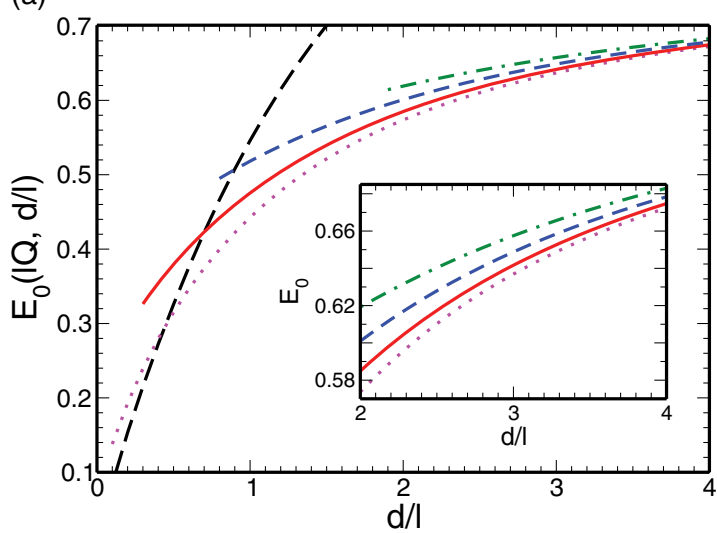

(b)

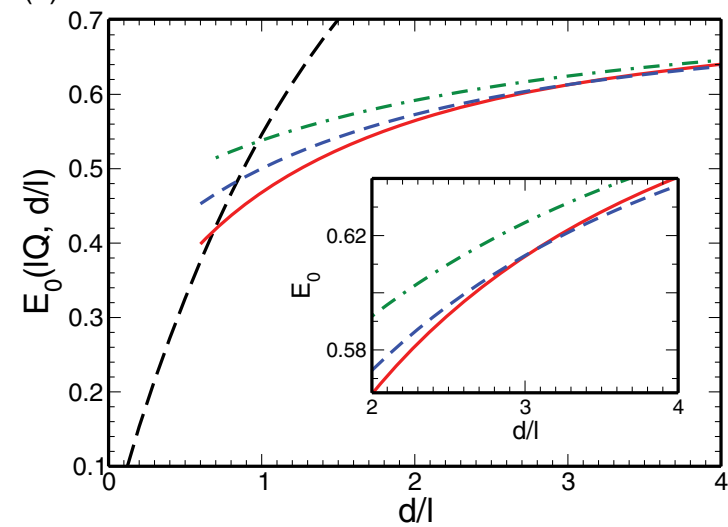

FIG. 4. (Color online) Ground-state energy per boson (in units of $e^{2} / \epsilon \ell$ ) as a function of $d / \ell$ : (a) one-mode approximation and (b) two-mode approximation. $\ell Q=0.5$ (dotted magenta line), 1 (solid red line), 1.5 (dashed blue line), and 2.0 (dotted-dashed green line). Long dashed black line: $Q=0$, one-mode approximation with $\mu_{0}=0$ (see text for details). Inset: details of the corresponding main plots focusing on the large $d / \ell$ region.

which yields

$$
\begin{aligned}
K & =K_{0}+\frac{1}{2} \sum_{\mathbf{q} \neq 0}\left(\Omega_{\mathbf{q}}^{+} a_{\mathbf{Q}+\mathbf{q}}^{\dagger} a_{\mathbf{Q}+\mathbf{q}}+\Omega_{\mathbf{q}}^{-} a_{\mathbf{Q}-\mathbf{q}} a_{\mathbf{Q}-\mathbf{q}}^{\dagger}\right) \\
& =\bar{K}_{0}+\sum_{\mathbf{q} \neq \mathbf{Q}} \bar{\Omega}_{\mathbf{q}} a_{\mathbf{q}}^{\dagger} a_{\mathbf{q}} .
\end{aligned}
$$

Here,

$$
\begin{aligned}
\Omega_{\mathbf{q}}^{ \pm} & = \pm \Delta_{\mathbf{q}}+\Omega_{\mathbf{q}}, \\
\Omega_{\mathbf{q}} & =\sqrt{\epsilon_{\mathbf{q}}^{2}-\lambda_{\mathbf{q}}^{2}}, \\
\epsilon_{\mathbf{q}} & =\frac{1}{2}\left(\epsilon_{\mathbf{q}}^{+}+\epsilon_{\mathbf{q}}^{-}\right), \quad \Delta_{\mathbf{q}}=\frac{1}{2}\left(\epsilon_{\mathbf{q}}^{+}-\epsilon_{\mathbf{q}}^{-}\right), \\
\bar{K}_{0} & =\left(\omega_{\mathbf{Q}}-\mu\right) N_{0}-\frac{1}{2} \sum_{\mathbf{q} \neq 0}\left(\epsilon_{\mathbf{q}}-\Omega_{\mathbf{q}}\right),
\end{aligned}
$$

the quasiparticle dispersion relation is given by

$$
\bar{\Omega}_{\mathbf{q}} \equiv \Omega_{\mathbf{q}-\mathbf{Q}}^{+},
$$

and the Bogoliubov coefficients obey

$$
u_{\mathbf{q}}^{2}=\frac{1}{2}+\frac{\epsilon_{\mathbf{q}}}{2 \Omega_{\mathbf{q}}}, \quad v_{\mathbf{q}}^{2}=-\frac{1}{2}+\frac{\epsilon_{\mathbf{q}}}{2 \Omega_{\mathbf{q}}}, \quad u_{\mathbf{q}} v_{\mathbf{q}}=\frac{\lambda_{\mathbf{q}}}{2 \Omega_{\mathbf{q}}} .
$$

The chemical potential $\mu$ can be obtained from the saddlepoint condition $\partial \bar{K}_{0} / \partial N_{0}=0$ : since $\partial \epsilon_{\mathbf{q}} / \partial N_{0}=\partial \lambda_{\mathbf{q}} / \partial N_{0}=$ $\lambda_{\mathbf{q}} / N_{0}$, one can show that

$$
\mu=\omega_{\mathbf{Q}}+\frac{1}{N_{0}} \sum_{\mathbf{q} \neq 0} \lambda_{\mathbf{q}} v_{\mathbf{q}}\left(v_{\mathbf{q}}-u_{\mathbf{q}}\right) \equiv \omega_{\mathbf{Q}}+\mu_{0} .
$$

$N_{0}$ follows from the conservation (on average) of the total number of bosons $N_{B}=\sum_{\mathbf{q}}\left\langle b_{\mathbf{q}}^{\dagger} b_{\mathbf{q}}\right\rangle=N_{\Phi} / 2=1 / 4 \pi \ell^{2}$ : from Eqs. (19), one finds that the relative number of bosons in the condensate is

$$
n_{0} \equiv \frac{N_{0}}{N_{B}}=1-\sum_{\mathbf{q} \neq 0} v_{\mathbf{q}}^{2}
$$

Finally, the ground-state energy $E_{0}(Q, d)=\bar{K}_{0}+\mu\langle\hat{N}\rangle$ reads as

$$
\frac{E_{0}(Q, d)}{N_{B}}=\frac{\bar{K}_{0}}{N_{B}}+\omega_{\mathbf{Q}}+\mu_{0}=\omega_{\mathbf{Q}}+\mu_{0}\left(1-n_{0}\right)-I_{01}
$$

with $I_{01}=\frac{1}{2 N_{B}} \sum_{\mathbf{q} \neq 0}\left(\epsilon_{\mathbf{q}}-\Omega_{\mathbf{q}}\right)$. Once $\mu$ and $n_{0}$ are known for fixed $\mathbf{Q}$ and $d / \ell$, the quasiparticle spectrum $\bar{\Omega}_{\mathbf{q}}$ and the ground-state energy (26) are completely determined.

\section{A. Zero-momentum BEC}

Before proceeding, we would like to briefly recall the results from our first analysis of the effective boson model (12) reported in Ref. 14.

By setting $\mathbf{Q}=0$ and $\mu_{0}=0$ in the above equations, we recover Eqs. (8) and (9) of Ref. 14. The choice $\mu_{0}=0$, based on the one-loop approximation, ${ }^{28}$ yields a gapless excitation spectrum for the zero-momentum BEC phase [see Fig. 2(a) from Ref. 14], in agreement with the Goldstone theorem.

We also find that the ground-state energy (26) increases with $d / \ell$ [Fig. 4 (long dashed black line)], and that the relative number of bosons in the condensate $n_{0}$ [Eq. (25)] decreases rather fast as $d / \ell$ increases [Fig. 5(a)]. Indeed, such a behavior of $n_{0}$ led us to include into the description the quartic terms in boson operators of the Hamiltonian (12) neglected in the Bogoliubov approximation. ${ }^{14}$ Considering these quartic terms in the so-called Popov approximation, ${ }^{29}$ we showed that the self-consistent equations admit solutions only for $d \leqslant d_{c 0}=0.4 \ell$. Here, we revisited the problem and perform more accurate numerical calculations. We find that $d_{c 0}=0.56 \ell$, which is even closer to the exact diagonalization estimates ${ }^{15,16}$ mentioned in the Introduction.

\section{B. Finite-momentum BEC}

Let us now consider $\ell Q \neq 0$ and discuss numerical solutions of Eqs. (24) and (25). It is possible to solve the self-consistent problem for all values of $\ell Q$ in the considered range as long as a finite (self-consistently calculated) $\mu_{0}$ is allowed and $d$ is larger than a minimum value $d_{\min }$. This feature 

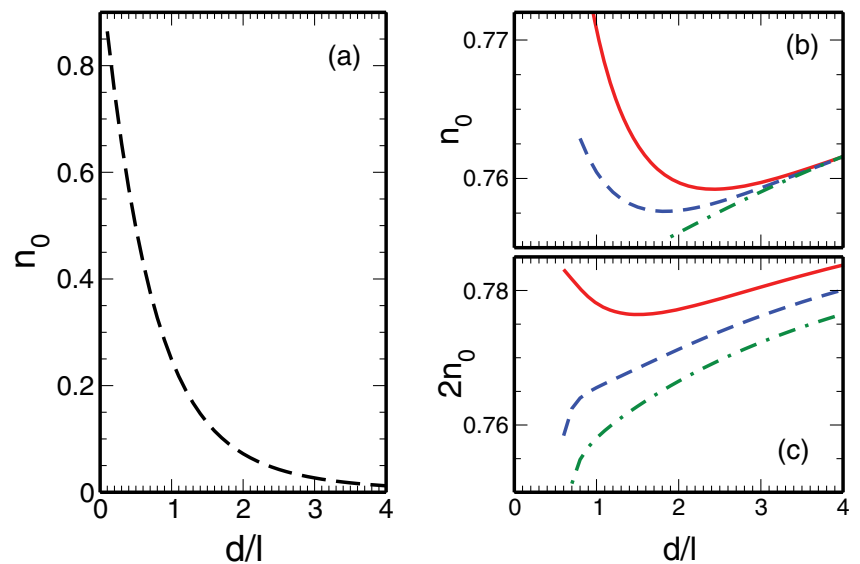

FIG. 5. (Color online) Relative number of bosons in the condensate $n_{0}$ [Eqs. (25) and (42)] as a function of $d / \ell$ : (a) zero-momentum BEC with $\mu_{0}=0$ (see text for details) and finite-momentum BEC with $\ell Q=1.0$ (solid red line), 1.5 (dashed blue line), and 2.0 (dotted-dashed green line) within the (b) one-mode approximation and (c) two-mode approximation.

is exemplified in Fig. 4(a), where we show the ground-state energy (26) as a function of $d / \ell$ for $\ell Q=0.5,1.0,1.5$, and 2.0. One can see that $d_{\min }=0.1,0.3,0.8$, and $1.9 \ell$, respectively, for $d=0.5,1.0,1.5$, and $2.0 \ell$. Note that the four configurations lie quite close in energy as $d / \ell$ increases, but the ground-state energy curves never cross each other. This behavior is also observed for all intermediate $\ell Q$ values (not shown here), i.e., $E_{0}(Q, d)$ increases with $\ell Q$ for a fixed $d / \ell$. It is clear that a finite-momentum BEC is lower in energy than the zero-momentum BEC discussed in the previous section for $d \gtrsim 1.0 \ell$. Interestingly, the $E_{0}(Q=0, d)$ and $E_{0}(Q \neq 0, d)$ curves cross at a (small) critical layer separation $d_{c 1}$, indicating that a first-order quantum phase transition from a zero-momentum BEC to a finite-momentum one takes place at this critical value. Note that for configurations with $0.5 \leqslant \ell Q \leqslant 1.0, d_{c 1}$ is within the range $0.45 \ell-0.7 \ell$, which includes the (updated) $d_{c 0}$ previously determined within the Popov approximation in Ref. 14.

One important consequence of a finite $\mu_{0}$ is that the dispersion relation of the (neutral) quasiparticles is now gapped. For instance, in Fig. 6, we show the excitation spectrum (22) along some particular momentum directions for the configuration with $\ell Q=1$ at $d=1.2 \ell$. The minimum gap $\Delta$ is at a momentum $\mathbf{q}_{\Delta}=-q_{\Delta} \hat{x}$, i.e., the angle between $\mathbf{q}_{\Delta}$ and $\mathbf{Q}$ is equal to $\pi$. For a fixed $\ell Q, q_{\Delta}$ continuously increases with $d / \ell$. We also find that, for a given $\ell Q$, the gap increases with $d / \ell$ as shown in Fig. 7 (dashed lines). The fact that a gap opens up at $d_{c 1}$ provides further support for a first-order quantum phase transition at this critical layer separation. Finally, note that $\bar{\Omega}_{\mathbf{q}}$ has no longer cylindrical symmetry $\bar{\Omega}_{\mathbf{q}} \neq \bar{\Omega}_{q}$, which differs from the excitation spectrum of the zero-momentum BEC [Fig. 2(a), Ref. 14]. This aspect and the peak in $\bar{\Omega}_{\mathbf{q}}$ at $\mathbf{q}=\mathbf{Q}$ are artifacts of the oversimplified one-mode approximation.

In order to understand the behavior of the excitation spectrum at small momentum q, we should look at the nature of the elementary excitations. Recall that a boson $b$ has an internal structure since it corresponds to an electron-hole pair.

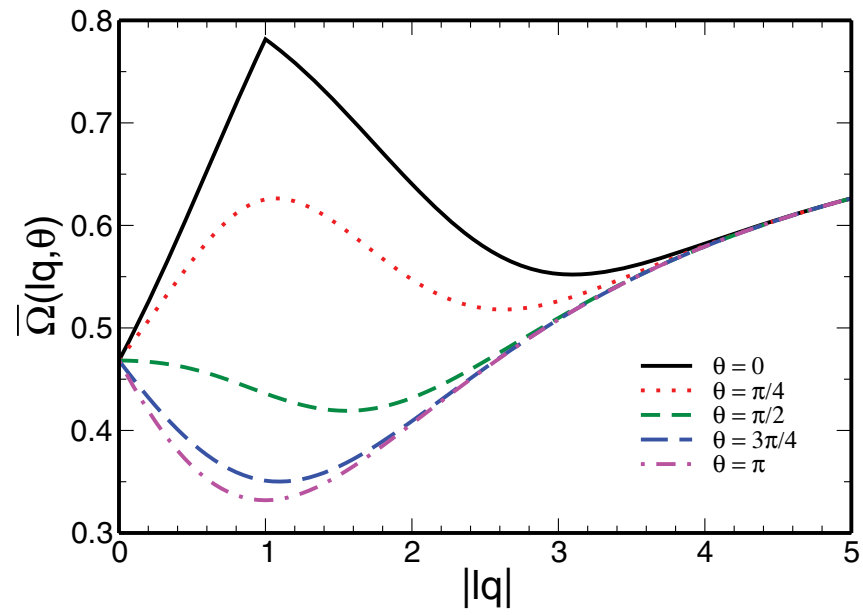

FIG. 6. (Color online) Dispersion relation of the (neutral) quasiparticles [Eq. (22)] (in units of $e^{2} / \epsilon \ell$ ) for a finite-momentum BEC with $\ell Q=1$ at $d=1.2 \ell$ along some particular momentum directions within the one-mode approximation.

An elementary excitation of the magnetic exciton BEC can be seen as an electron-hole pair with momentum $\mathbf{Q}$ which is taken from the condensate, broken and recombined again in a electron-hole pair, but now with a momentum $\mathbf{q} \neq \mathbf{Q}$. Apart from the corrections due to the boson-boson interaction potential [Eq. (14)], the excitation energy $\bar{\Omega}_{\mathbf{q}}$ is related to the difference $\Delta E_{b}$ between the binding energies of the pairs with momentum $\mathbf{Q}$ and $\mathbf{q}$, namely,

$$
\bar{\Omega}_{\mathbf{q}} \sim \Delta E_{b}=\omega_{\mathbf{Q}}-\omega_{\mathbf{q}},
$$

where $\omega_{\mathbf{q}}$ is the dispersion relation of the free bosons [Eq. (13)]. Let us first consider the zero-momentum BEC. In this case, the bosons are condensed in the lowest single-particle energy mode $\mathbf{Q}=0$, and therefore $\lim _{\mathbf{q} \rightarrow 0}\left(\omega_{\mathbf{Q}}-\omega_{\mathbf{q}}\right)=0$ which yields a gapless excitation spectrum, i.e., the system displays a Goldstone mode. On the other hand, in a finitemomentum BEC, the bosons are not condensed in the lowest single-particle energy mode. This is an important feature

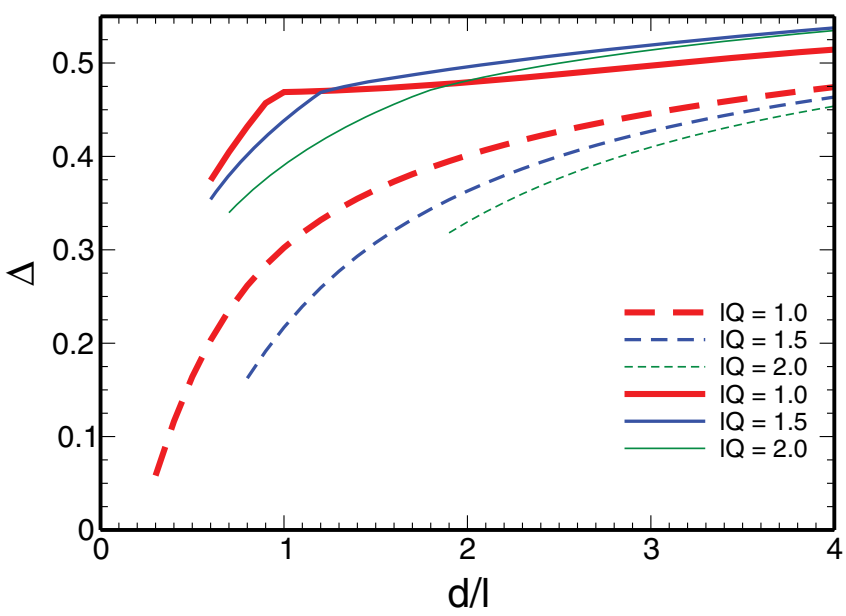

FIG. 7. (Color online) Minimum gap energy $\Delta$ (in units of $e^{2} / \epsilon \ell$ ) of the (neutral) quasiparticle excitations as a function of $d / \ell$ for a finite-momentum BEC with $\ell Q=1,1.5$, and 2. Dashed lines: onemode approximation; solid lines: two-mode approximation. 
which implies that $\lim _{\mathbf{q} \rightarrow 0}\left(\omega_{\mathbf{Q}}-\omega_{\mathbf{q}}\right) \neq 0$, i.e., the Goldstone mode disappears. In other words, the internal structure of the boson $b$ combined with a macroscopic occupation of a higher-energy single-particle mode leads to the disappearance of the Goldstone mode. Such a behavior reminds us of the excitation spectrum of a BCS superconductor. ${ }^{30}$ We will return to this issue in Sec. VIA.

Finally, we find that the relative number of bosons in the condensate $n_{0}$ [Eq. (25)] is roughly independent of $d / \ell$ and close to one. Such an aspect, illustrated in Fig. 5(b), is related to the existence of a finite excitation gap, which reduces quantum fluctuation effects compared with a gapless case (the zero-momentum BEC). The fact that $n_{0} \approx 1$ tells us that the Bogoliubov approximation is indeed quite reasonable to study a finite-momentum BEC phase, in contrast with the zero-momentum BEC, which requires a more involved approximation.

\section{TWO-MODE APPROXIMATION}

So far, we have considered that the bosons $b$ condense in just one particular single-particle mode $\mathbf{q}=Q \hat{x}$. As mentioned in the previous section, since the single-particle boson dispersion relation (13) has cylindrical symmetry, $\omega_{\mathbf{q}}=\omega_{q}$, the bosons $b$ could, in principle, condense in more than one mode $\mathbf{q}$ provided that $q=Q$. In this section, we discuss such a possibility, in particular, we assume that the BEC is split into two pieces: both $\mathbf{q}= \pm \mathbf{Q}$ modes, with $\mathbf{Q}=Q \hat{x}$ and $\ell Q \neq 0$, are now macroscopically occupied. Again, the Bogoliubov approximation is employed to analyze the effective boson model (12). We hereafter denote such a scheme two-mode approximation.

Here, we basically follow the lines of Sec. III and start by performing the substitutions

$$
\begin{gathered}
b_{\mathbf{Q}}^{\dagger}=b_{\mathbf{Q}}=\left\langle b_{\mathbf{Q}}^{\dagger}\right\rangle=\left\langle b_{\mathbf{Q}}\right\rangle \rightarrow \sqrt{N_{0}}, \\
b_{-\mathbf{Q}}^{\dagger}=b_{-\mathbf{Q}}=\left\langle b_{-\mathbf{Q}}^{\dagger}\right\rangle=\left\langle b_{-\mathbf{Q}}\right\rangle \rightarrow \sqrt{\bar{N}_{0}}
\end{gathered}
$$

in Eq. (15). The equivalent of Eq. (17) is now given by Eq. (C1) (see Appendix C). In order to diagonalize the Hamiltonian (C1), it is useful to introduce the four-component vector

$$
\Psi_{\mathbf{q}}^{\dagger}=\left(b_{\mathbf{Q}+\mathbf{q}}^{\dagger} b_{-\mathbf{Q}+\mathbf{q}}^{\dagger} b_{-\mathbf{Q}-\mathbf{q}} b_{\mathbf{Q}-\mathbf{q}}\right) .
$$

Equation $(\mathrm{C} 1)$ can then be expressed in matrix form

$$
K=K_{0}+\frac{1}{4} \sum_{\mathbf{q}} \Psi_{\mathbf{q}}^{\dagger} \hat{H}_{\mathbf{q}} \Psi_{\mathbf{q}}
$$

where the $4 \times 4$ matrix $\hat{H}_{\mathbf{q}}$ reads as

$$
\hat{H}_{\mathbf{q}}=\left(\begin{array}{cc}
\hat{A}_{\mathbf{q}} & \hat{B}_{\mathbf{q}} \\
\hat{B}_{\mathbf{q}} & \hat{A}_{\mathbf{q}}
\end{array}\right)
$$

with the $2 \times 2$ matrices $\hat{A}_{\mathbf{q}}$ and $\hat{B}_{\mathbf{q}}$ given by

$$
\hat{A}_{\mathbf{q}}=\left(\begin{array}{cc}
\epsilon_{\mathbf{q}}+\Delta_{\mathbf{q}} & \gamma_{\mathbf{q}} \\
\gamma_{\mathbf{q}} & \epsilon_{\mathbf{q}}-\Delta_{\mathbf{q}}
\end{array}\right) \quad \text { and } \quad \hat{B}_{\mathbf{q}}=\left(\begin{array}{cc}
\xi_{\mathbf{q}} & \lambda_{\mathbf{q}} \\
\lambda_{\mathbf{q}} & \xi_{\mathbf{q}}
\end{array}\right) \text {. }
$$

Here, we assume that both condensates have the same number of bosons and set $\bar{N}_{0}=N_{0}$. The coefficients $\epsilon_{\mathbf{q}}$ and $\Delta_{\mathbf{q}}$ are defined in Eq. (21), while $\gamma_{\mathbf{q}}, \xi_{\mathbf{q}}$, and $\lambda_{\mathbf{q}}$ are shown in Appendix C [see Eqs. (C2) and (C3)].
The diagonalization of the $4 \times 4$ problem (29) is more involved than the $2 \times 2$ one corresponding to Eq. (17). Therefore, it is more convenient here to use the procedure described in Ref. 31: Since we are dealing with a bosonic system, instead of $\hat{H}_{\mathbf{q}}$, one should diagonalize

$$
\hat{I}_{B} \hat{H}_{\mathbf{q}} \quad \text { with } \quad \hat{I}_{B}=\left(\begin{array}{cc}
\hat{I} & 0 \\
0 & -\hat{I}
\end{array}\right) .
$$

The (positive) eigenvalues of the matrix (31) are

$$
\Omega_{\mathbf{q}}^{ \pm}=\sqrt{C_{\mathbf{q}} \pm 2 D_{\mathbf{q}}}
$$

where

$$
\begin{aligned}
& C_{q}=\epsilon_{\mathbf{q}}^{2}+\Delta_{\mathbf{q}}^{2}+\gamma_{\mathbf{q}}^{2}-\lambda_{\mathbf{q}}^{2}-\xi_{\mathbf{q}}^{2}, \\
& D_{q}=\left[\Delta_{\mathbf{q}}^{2}\left(\epsilon_{\mathbf{q}}^{2}-\lambda_{\mathbf{q}}^{2}\right)+\left(\gamma_{\mathbf{q}} \epsilon_{\mathbf{q}}-\lambda_{\mathbf{q}} \xi_{\mathbf{q}}\right)^{2}\right]^{1 / 2} .
\end{aligned}
$$

Equation (29) then acquires the form

$$
K=K_{0}+\frac{1}{4} \sum_{\mathbf{q}} \Phi_{\mathbf{q}}^{\dagger} \hat{H}_{\mathbf{q}}^{\prime} \Phi_{\mathbf{q}}
$$

where the $4 \times 4$ matrix $\hat{H}_{\mathbf{q}}^{\prime}$ reads as

$$
\hat{H}_{\mathbf{q}}^{\prime}=\left(\begin{array}{cc}
\hat{h}_{\mathbf{q}} & 0 \\
0 & \hat{h}_{\mathbf{q}}
\end{array}\right) \quad \text { with } \quad \hat{h}_{\mathbf{q}}=\left(\begin{array}{cc}
\Omega_{\mathbf{q}}^{+} & 0 \\
0 & \Omega_{\mathbf{q}}^{-}
\end{array}\right)
$$

and the new four-component vector $\Phi_{\mathbf{q}}^{\dagger}$ is given by

$$
\Phi_{\mathbf{q}}^{\dagger}=\left(a_{\mathbf{Q}+\mathbf{q}}^{\dagger} a_{-\mathbf{Q}+\mathbf{q}}^{\dagger} a_{-\mathbf{Q}-\mathbf{q}} a_{\mathbf{Q}-\mathbf{q}}\right) .
$$

The relation between the two sets of bosonic operators $a_{ \pm \mathbf{Q} \pm \mathbf{q}}$ and $b_{ \pm \mathbf{Q} \pm \mathbf{q}}$ is

$$
\Phi_{\mathbf{q}}=\hat{M}_{\mathbf{q}} \Psi_{\mathbf{q}}, \quad \text { where } \quad \hat{M}_{\mathbf{q}}=\left(\begin{array}{cc}
\hat{U}_{\mathbf{q}} & \hat{V}_{\mathbf{q}} \\
\hat{V}_{\mathbf{q}} & \hat{U}_{\mathbf{q}}
\end{array}\right)
$$

with $\hat{U}_{\mathbf{q}}$ and $\hat{V}_{\mathbf{q}}$ being $2 \times 2$ matrices

$$
\hat{U}_{\mathbf{q}}=\left(\begin{array}{ll}
u_{1}(\mathbf{q}) & u_{3}(\mathbf{q}) \\
u_{2}(\mathbf{q}) & u_{4}(\mathbf{q})
\end{array}\right), \quad \hat{V}_{\mathbf{q}}=\left(\begin{array}{ll}
v_{1}(\mathbf{q}) & v_{3}(\mathbf{q}) \\
v_{2}(\mathbf{q}) & v_{4}(\mathbf{q})
\end{array}\right),
$$

the elements of which are the Bogoliubov coefficients. The complete expressions of the Bogoliubov coefficients $u_{i}(\mathbf{q})$ and $v_{i}(\mathbf{q})$ are quite long and they can be found in Appendix C.

Equation (34) can be rewritten as

$$
K=\bar{K}_{0}+\sum_{\mathbf{q} \neq \pm \mathbf{Q}} \bar{\Omega}_{\mathbf{q}} a_{\mathbf{q}}^{\dagger} a_{\mathbf{q}}
$$

where the quasiparticle energy $\bar{\Omega}_{\mathbf{q}}$ reads as

$$
\bar{\Omega}_{\mathbf{q}}=\frac{1}{4}\left(\Omega_{\mathbf{Q}+\mathbf{q}}^{+}+\Omega_{-\mathbf{Q}+\mathbf{q}}^{+}+\Omega_{\mathbf{Q}+\mathbf{q}}^{-}+\Omega_{-\mathbf{Q}+\mathbf{q}}^{-}\right)
$$

and

$$
\begin{aligned}
\bar{K}_{0}= & 2 N_{0}^{2} v_{2 \mathbf{Q}}(\mathbf{Q}, \mathbf{Q})+2 N_{0}\left(\omega_{\mathbf{Q}}-\mu\right) \\
& +\frac{1}{4} \sum_{\mathbf{q}}\left(\Omega_{\mathbf{q}}^{+}+\Omega_{\mathbf{q}}^{-}-\epsilon_{\mathbf{q}}^{+}-\epsilon_{\mathbf{q}}^{-}\right) .
\end{aligned}
$$

Again, from the saddle-point condition $\partial \bar{K}_{0} / \partial N_{0}=0$, the chemical potential $\mu$ can be calculated: since from Eqs. (C2) 
with $\bar{N}_{0}=N_{0}$ we have

$$
\begin{gathered}
\partial \epsilon_{\mathbf{q}}^{ \pm} / \partial N_{0}=\partial \lambda_{\mathbf{q}} / \partial N_{0}=\lambda_{\mathbf{q}} / N_{0}, \quad \partial \Delta_{\mathbf{q}} / \partial N_{0}=0, \\
\partial \gamma_{\mathbf{q}} / \partial N_{0}=\gamma_{\mathbf{q}} / N_{0}, \quad \partial \xi_{\mathbf{q}} / \partial N_{0}=\xi_{\mathbf{q}} / N_{0},
\end{gathered}
$$

after some algebra, we find that

$$
\mu=\omega_{\mathbf{Q}}+\mu_{0}+2 N_{0} v_{2 \mathbf{Q}}(\mathbf{Q}, \mathbf{Q}) \equiv \omega_{\mathbf{Q}}+\mu_{0}+\mu_{1} .
$$

The quantity $\mu_{0}$ [see Eq. (C4)] is different from the one-mode approximation expression (24). From the conservation (on average) of the total number of bosons $N_{B}=\sum_{\mathbf{q}}\left\langle b_{\mathbf{q}}^{\dagger} b_{\mathbf{q}}\right\rangle=$ $2 N_{0}+\sum_{\mathbf{q} \neq \pm \mathbf{Q}}\left\langle b_{\mathbf{q}}^{\dagger} b_{\mathbf{q}}\right\rangle$, it follows that the relative number of bosons in the condensate $n_{0}$ is given by

$$
n_{0} \equiv \frac{N_{0}}{N_{B}}=\frac{1}{2}-\frac{1}{4} \sum_{i=1}^{4} \sum_{\mathbf{q}} v_{i}^{2}(\mathbf{q}) .
$$

Finally, it is easy to see that the ground-state energy $E_{0}(Q, d)=$ $\bar{K}_{0}+\mu\langle\hat{N}\rangle$ reads as

$$
\begin{aligned}
\frac{E_{0}(Q, d)}{N_{B}} & =\frac{\bar{K}_{0}}{N_{B}}+\omega_{\mathbf{Q}}+\mu_{0}+\mu_{1} \\
& =\omega_{\mathbf{Q}}+\mu_{0}\left(1-2 n_{0}\right)+\mu_{1}\left(1-n_{0}\right)-I_{02}
\end{aligned}
$$

with $I_{02}=\frac{1}{4 N_{B}} \sum_{\mathbf{q}}\left(\epsilon_{\mathbf{q}}^{+}+\epsilon_{\mathbf{q}}^{-}-\Omega_{\mathbf{q}}^{+}-\Omega_{\mathbf{q}}^{-}\right)$. Similarly to the one-mode approximation, Sec. III, we numerically solve the self-consistent equations (41) and (42) and determine $n_{0}$ and $\mu$ for fixed $\ell Q$ and $d / \ell$.

In Fig. 4(b), we plot the ground-state energy (43) as a function of $d / \ell$ for three different configurations, those with $\ell Q=1.0,1.5$, and 2.0. Likewise the one-mode approximation, the self-consistent equations can be solved only for $d$ larger than a minimum value $d_{\min }$. However, we now have $d_{\min }=0.6$, 0.6 , and $0.7 \ell$, respectively, for $\ell Q=1.0,1.5$, and 2.0, which differ from the one-mode approximation results. Comparing the ground-state energies obtained with both one- and twomode approximations for a given $\ell Q$, we clearly see that the latter is lower than the former: since $\omega_{\mathbf{q}}=\omega_{q}$, macroscopic occupation of both $\pm \mathbf{Q}$ modes are equally likely. The system then profits from this fact by splitting the condensate into $n=2$ equal pieces, binding them and lowering the total energy. Again, a finite-momentum BEC is more favorable than a zeromomentum one for $d \gtrsim 1.0 \ell$ and the $E_{0}(Q \neq 0, d)$ and the $E_{0}(Q=0, d)$ (one-mode approximation with $\mu_{0}=0$ ) curves cross at small $d$. In particular, $E_{0}(\ell Q=1, d)$ and $E_{0}(Q=$ $0, d)$ cross at $d_{c 1} \approx 0.68 \ell$, in good agreement with the result derived in the previous section. Therefore, both one- and twomode approximations indicate that a first-order quantum phase transition may occur at small $d / \ell$.

Concerning the large $d$ region, we again find that the configurations with $\ell Q \neq 0$ are quite close in energy but now, differently from the one-mode approximation, the different $E_{0}(Q \neq 0, d)$ curves cross each other. For instance, $E_{0}(\ell Q=1, d)$ and $E_{0}(\ell Q=1.5, d)$ cross at $d \approx 3.1 \ell$ [see inset Fig. 4(b)]. Indeed, we find several crossings between the different ground-state energy curves for $1.0 \leqslant \ell Q \leqslant 2.0$ and $0.7 \ell \leqslant d \leqslant 4 \ell$. In particular, the magnitude of the momentum $\left|\ell \mathbf{Q}_{0}\right|$ corresponding to the lowest-energy configuration for a given $d / \ell$ is shown in Fig. 8. The fact that $\ell Q_{0}$ increases with $d / \ell$ corroborates the scenario proposed in Sec. IIC that the larger $\ell Q$, the lower the correlation between the two

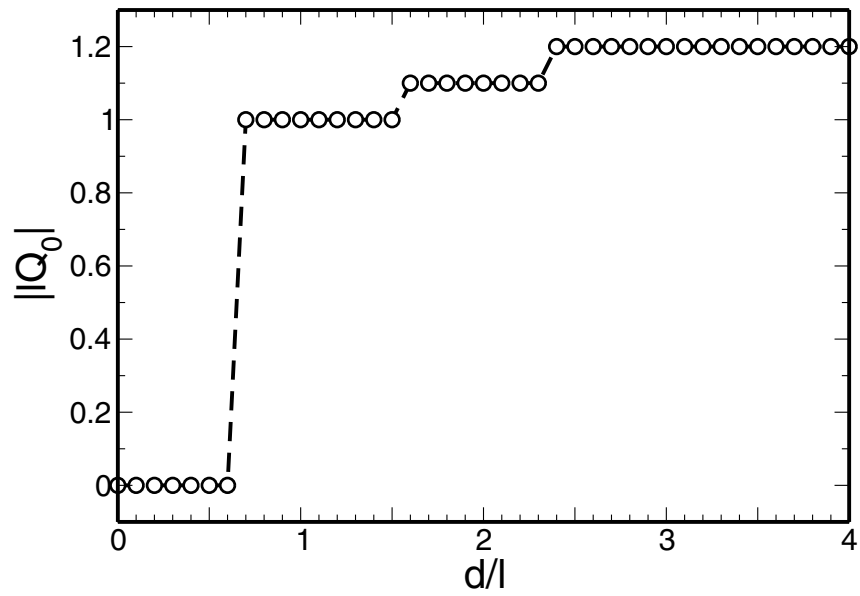

FIG. 8. Magnitude of the momentum $\ell \mathbf{Q}_{0}$ corresponding to the lowest-energy configuration for a fixed $d / \ell$, two-mode approximation. Dashed line is a guide to the eyes.

layers. Note that the one-mode approximation is not enough to capture such a behavior. Moreover, the results also indicate that another first-order quantum phase transition may take place at larger $d$, from one finite-momentum BEC with small $\ell Q_{0}$ to another one with a larger $\ell Q_{0}$. In particular, the transition $\ell Q_{0}=1.0 \rightarrow \ell Q_{0}=1.1$ occurs at $d_{c 2}=1.6 \ell$, which is very close to the critical layer separation where the incompressiblecompressible phase transition is experimentally observed. ${ }^{3}$ Finally, we should mention that solutions for the $\ell Q=0.8$ and 0.9 configurations are also possible but, since they are very close in energy to the $\ell Q=1.0$ configuration, we decided to neglect them in the above discussion.

Differently from the one-mode approximation, here the inversion symmetry of the excitation spectrum $\bar{\Omega}_{\mathbf{q}}$ [Eq. (39)] is preserved as exemplified in Fig. 9 for the

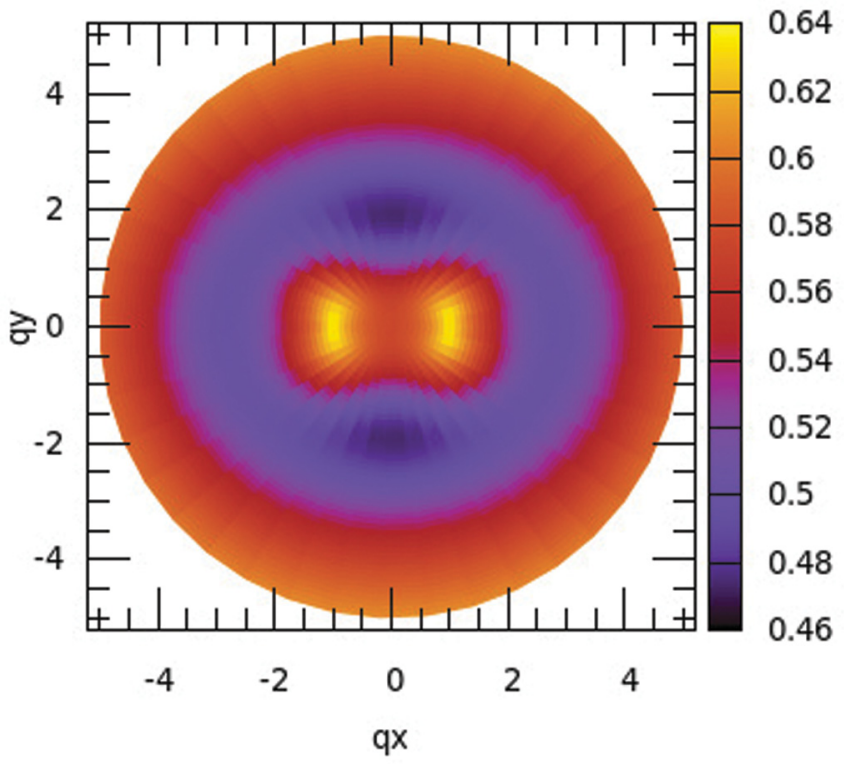

FIG. 9. (Color online) Contour plot of the quasiparticle dispersion relation $\bar{\Omega}_{\mathbf{q}}$ [Eq. (39)] (in units of $e^{2} / \epsilon \ell$ ) for a finite-momentum BEC with $\ell Q=1$ at $d=1.5 \ell$, two-mode approximation. 

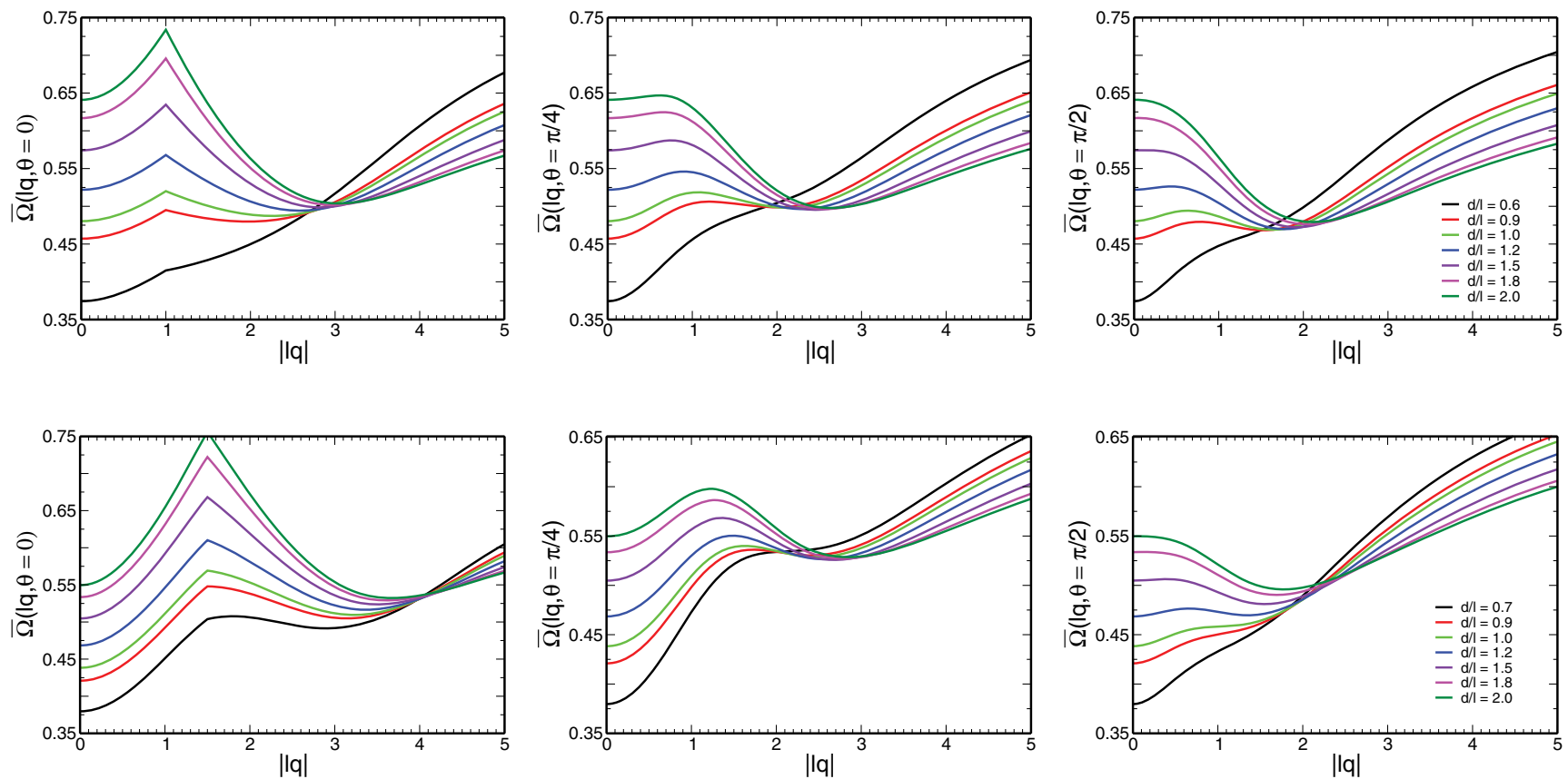

FIG. 10. (Color online) Quasiparticle dispersion relation $\bar{\Omega}_{\mathbf{q}}$ [Eq. (39)] (in units of $e^{2} / \epsilon \ell$ ) along some particular momentum directions for several values of the ratio $d / \ell$. Finite-momentum BEC with $\ell Q=1.0$ (upper row) and $\ell Q=1.5$ (lower row), two-mode approximation ( $d / \ell$ increases from bottom to top at $\ell q=0$ ).

finite-momentum BEC with $\ell Q=1.0$ at $d=1.5 \ell$. Again, a finite (self-consistently determined) $\mu_{0}$ leads to a gapped excitation spectrum. Note that the minimum gap, which is larger than the corresponding one determined within the one-mode approximation, increases with $d / \ell$ for a fixed $\ell Q$ [Fig. 7 (solid lines)]. Interestingly, for small $d / \ell$, the minimum gap is located at the origin $\left(\mathbf{q}_{\Delta}=0\right)$ but, as $d / \ell$ increases, the position of the minimum gap abruptly changes to $\mathbf{q}_{\Delta}=q_{\Delta} \hat{y}$ (the angle between $\mathbf{q}_{\Delta}$ and $\mathbf{Q}$ is now $\pi / 2$, in contrast with the one-mode-approximation result) and then $\ell q_{\Delta}$ continuously increases with $d / \ell$. Such a behavior is exemplified in Fig. 10 for the $\ell Q=1.0$ (upper row) and 1.5 (lower row) configurations. Also, the kinks observed in Fig. 7 (solid lines) are signatures of this abrupt change in $\mathbf{q}_{\Delta}$. Finally, some words about the singularities of the excitation spectrum are here in order: we believe that the peaks in $\bar{\Omega}_{\mathbf{q}}$ at $\pm \mathbf{Q}$ (also found in the one-mode approximation) might be an artifact of the two-mode approximation and that they may disappear as we increase the number of components $n$ (even) of the finite-momentum condensate. We will return to this point in Sec. VIB.

Concerning the relative number of bosons in the two condensate pieces $2 n_{0}$ [Fig. 5(c)], we can see that its behavior is similar to the one found in the previous section: it is almost independent of $d / \ell$ and $2 n_{0}$ is close to one. The latter indicates that the Bogoliubov approximation is indeed appropriate to study the finite-momentum BEC even if the condensate is split into more than one piece.

As a final remark, we would like to comment on the fact that the results found in this section seem to contradict Nozières, ${ }^{32}$ who argued that a fragmentation of the condensate into two pieces costs a macroscopic extensive exchange energy and therefore it is not favorable. A careful analysis shows that there is no contradiction. Let us denote $E_{01}$ and $E_{02}$ the ground-state energy, respectively, obtained within the one-mode [Eq. (26)] and two-mode [Eq. (43)] approximations. Using the fact that $n_{0}$ [one-mode approximation, Eq. (25)] is roughly equal to $2 n_{0}$ [two-mode approximation, Eq. (42)], we have

$$
E_{02}-E_{01} \approx \mu_{1}\left(1-n_{0}\right)-I_{02}+I_{01} .
$$

Comparing the above equation with Eq. (4) from Ref. 32, we realize that Nozières considerations only take into account the first term in the above equation and completely neglect the other terms which, as we have seen, provide important corrections. In particular, for the bilayer QHS, symmetry considerations also indicate that a BEC split into two parts has lower energy than a single condensate: recall that the excitation spectrum obtained within the two-mode approximation is more symmetrical than the one derived in the one-mode approximation.

\section{PROPERTIES OF A BEC OF MAGNETIC EXCITONS}

It is easy to see from Eq. (10) that

$$
\langle\rho(\mathbf{k})\rangle=\delta_{k, 0} N_{\Phi},
$$

which is valid for both one- and two-mode approximations regardless the value of $\ell Q$. Equation (44) implies that, in principle, the $\mathrm{BEC}$ of magnetic excitons is a homogeneous phase (see discussion at the end of the section). Concerning the expectation value of the $\hat{z}$ component of the pseudospin density operator (11), one shows (two-mode approximation)

$$
\left\langle S_{z}(\mathbf{k})\right\rangle=\sqrt{N_{0} \bar{N}_{0}} \exp \left(-|\ell Q|^{2} / 4\right)\left(\delta_{\mathbf{k},-2 \mathbf{Q}}+\delta_{\mathbf{k}, 2 \mathbf{Q}}\right) .
$$


Note that (45) vanishes within the one-mode approximation since $\bar{N}_{0} \rightarrow 0$.

Further insight into a BEC of magnetic excitons can be obtained by looking at the pair correlation function, which is defined as ${ }^{33}$

$$
g(\mathbf{r})-1=\frac{1}{N} \sum_{\mathbf{q}} e^{-i \mathbf{q} \cdot \mathbf{r}}[S(\mathbf{q})-1],
$$

where the static structure factor is given by

$$
S(\mathbf{q})=\frac{1}{N}\langle\rho(-\mathbf{q}) \rho(\mathbf{q})\rangle-N \delta_{q, 0}
$$

with $\rho(\mathbf{q})$ being the Fourier transform of the electron density operator. The pair correlation function basically tells us the probability of finding an electron at the position $\mathbf{r}$ given that there is another one at the origin. The analytical expression of $g(\mathbf{r})$, both at the one- and two-mode approximations, is quite lengthy and can be found in Appendix D. Here, we just comment on its numerical evaluation.

In Fig. 11, we plot the pair correlation function along one particular $\mathbf{r}$ direction for the zero-momentum BEC (one-mode approximation with $\mu_{0}=0$ ) and for the finitemomentum BECs with $\ell Q=1$ and 2 at $d=1.0,1.5$, and $2.0 \ell$, calculated in the two-mode approximation. Within the approximations considered here, $g(\mathbf{r})$ is $d / \ell$ independent for the zero-momentum BEC (see dashed line in Fig. 11). In this case, the pair correlation function vanishes as $r \rightarrow 0$, indicating the existence of a correlation hole around the electron, and it is constant at large $r$, the same features displayed by the single-layer QHS at $v=1 .^{34} \mathrm{~A}$ distinct behavior is found for the finite-momentum BEC. Note that now $g(0) \neq 0$, indicating that two electrons (with different pseudospins quantum numbers) can be very close to each other, corroborating somehow the schematic picture for a finite-momentum BEC depicted in Fig. 1(c). Moreover, small oscillations at large $r / \ell$ are observed, which are characteristic of a composite fermion Fermi liquid. ${ }^{34}$ These findings support the proposal that the ground state of the bilayer QHS in the intermediate $d / \ell$ region can be described by a finitemomentum BEC of bosons $b$.

As a final remark, we would like to mention that in Ref. 35 it is shown that an exciton condensate has diagonal long-range order. Interestingly, the average value of the density operator is constant and only the density-density correlation function has Fourier components of the type $\exp \left[-i \mathbf{K} \cdot\left(\mathbf{r}_{1}-\mathbf{r}_{2}\right)\right]$. Therefore, based only on Eq. (44), we would expect that the finite-momentum $\mathrm{BEC}$ of magnetic excitons corresponds to an inhomogeneous phase. However, since the pair correlation function (46), which is related to the density-density correlation function (47), displays a behavior characteristic of a liquid, we then conclude that the finite-momentum BEC of magnetic excitons is indeed a homogeneous phase. The disagreement between our results and the general analysis of Ref. 35 might be related to the fact that here the electrons are restricted to the lowest Landau level. Recall that such a restriction, e.g., modifies the commutation relations between the electron and pseudospin density operators. ${ }^{36}$

\section{DISCUSSION}

\section{A. Relation to previous work}

In this section, we briefly summarize some previous results about the bilayer QHS and compare them with the ones derived here using the one- and two-mode approximations.

Park ${ }^{21}$ proposed that the bilayer QHS at $v_{T}=1$ develops a pseudospin spiral long-range order at intermediate $d / \ell$ values. In this case, the main interlayer correlations are established between electrons and holes localized in different guiding centers [see Eq. (8), Ref. 21]. Interestingly, the excitation spectrum is gapped. It is argued that there is no fundamental reason for a Goldstone mode in this case (see note 14 in Ref. 21). These two aspects above discussed suggest that the pseudospin spiral state bears some similarities with the finite-momentum BEC of bosons $b$. Moreover, it is also conjectured ${ }^{21}$ that the ground state is indeed given by a bound state between two pseudospin spirals with opposite winding direction. Recall that by splitting the finite-momentum condensate into two equal pieces, the total energy of the system decreases (see Fig. 4).

The ground-state energy of finite-size systems was calculated within the exact diagonalization technique. ${ }^{20}$ It is shown that, regardless the size of the system, the ground-state energy is almost constant for large $d$, a signature of the decoupling between the two layers. As we can see in Fig. 4, the ground-state energy of a finite-momentum BEC slowly varies for larger $d / \ell$. Moreover, the variation decreases when the condensate is separated into two equal pieces.

Nomura and Yoshioka also consider finite-size systems and calculate the pair correlation function (46) via exact diagonalization. ${ }^{19}$ It is found that for $d=0.3 \ell$, both $g_{\uparrow \uparrow}(\mathbf{r})$ and $g_{\uparrow \downarrow}(\mathbf{r})$ vanish as $r \rightarrow 0$ but, for a larger $d=0.9 \ell$, while $g_{\uparrow \uparrow}(0)$ vanishes, $g_{\uparrow \downarrow}(0)$ is now finite. Concerning the large $r$ region, both $g_{\uparrow \uparrow}(\mathbf{r})$ and $g_{\uparrow \downarrow}(\mathbf{r})$ seem to be constant for $d=0.3 \ell$ but, for $d=0.9 \ell$, they show small oscillations. Note that the pair correlation function (Fig. 11) qualitatively displays the same features.

Based on a Chern-Simons gauge theory, Bonesteel et al. ${ }^{37}$ show that by approaching two composite fermion Fermi seas, there is always an instability towards the formation of composite fermion Cooper pairs. The theory is valid only in the large $d$ region. The possibility of interlayer composite fermion pairing is considered in Ref. 18 where some trial wave functions are discussed. Assuming a $p_{x}-i p_{y}$ pairing instability, it is shown that the two possible wave functions correspond to the $(3,3,-1)$ and the so-called "strong" pairing (SP) states. The former phase displays a gapped (neutral) excitation spectrum. A qualitative phase diagram is also proposed and one of the possibilities is that the ground state changes as $d / \ell$ increases according to the following sequence: 111-SP- $(3,3,-1)$ state. Unfortunately, it is not clear how to compare a finite-momentum BEC of magnetic excitons with the SP and $(3,3,-1)$ states.

Further support for pairing between interlayer composite fermions is provided in Ref. 16. However, the numerical results indicate that a $p_{x}+i p_{y}$ pairing may occur instead of the $p_{x}-i p_{y}$ considered by Kim et al. ${ }^{18}$ Mixed Fermi-Bose trial wave functions were then proposed $^{6}$ to describe the intermediate $d \sim \ell$ region, where the bosonic part is given by the 111 state, while the fermionic one is given by a paired 
(a)

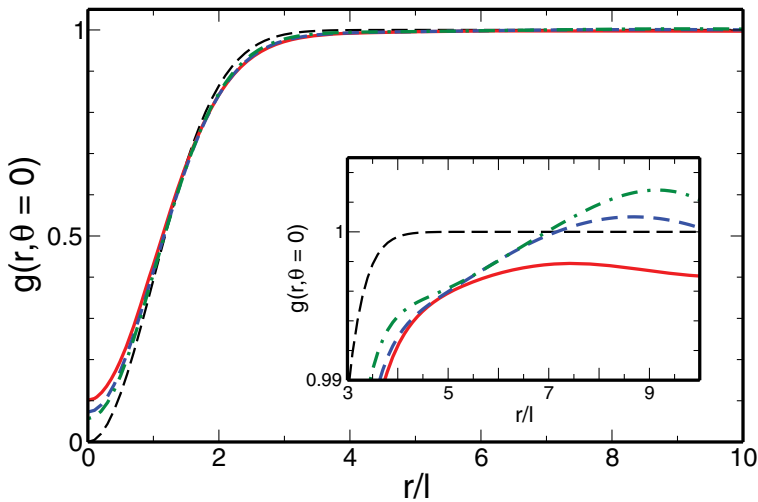

(b)

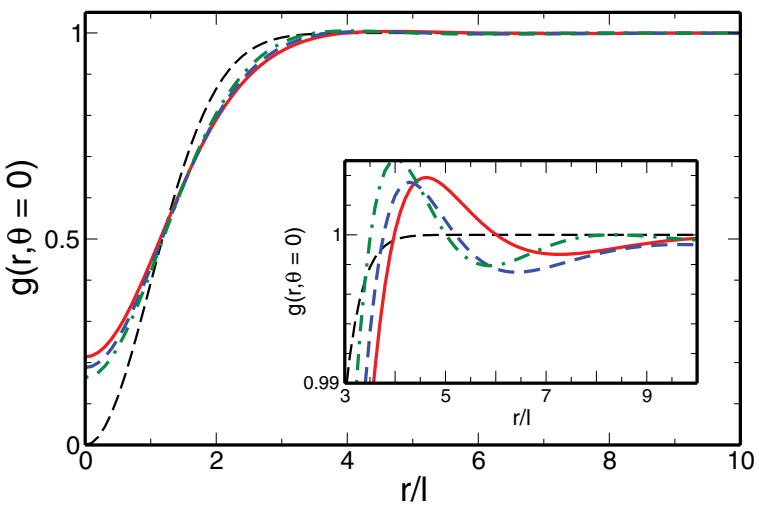

FIG. 11. (Color online) Pair correlation function (46) along one particular $\mathbf{r}$ direction. Dashed black line: zero-momentum BEC, one-mode approximation with $\mu_{0}=0$ [Eq. (D5)]. Finite-momentum BEC with (a) $\ell Q=1.0$ and (b) $\ell Q=2.0$, two-mode approximation. $d=1$ (solid red line), 1.5 (long dashed blue line), and $2 \ell$ (dotted-dashed green line). Insets: details of the corresponding main plots showing the behavior of the pair correlation function in the large $r / \ell$ region.

composite fermion state. Such an approach indeed follows the lines of an earlier work by Simon and co-workers, ${ }^{15}$ where mixed wave functions are considered, but here the fermionic piece is given by two composite fermion Fermi seas. It is shown that $^{6}$ the mixed wave functions with paired states provided a better description for the intermediate $d \sim \ell$ region than the ones which do not include pairing. Again, it is difficult to compare this mixed Fermi-Bose wave functions with the finite-momentum BEC discussed here. We would like to point out that the description in terms of a finite-momentum BEC of magnetic excitons involves only bosonic degrees of freedom.

Finally, studying the bilayer QHS within a GinzburgLandau theory, Ye and Jiang ${ }^{8}$ suggested that the ground state is given by a pseudospin density wave for $d_{c 1}<d<d_{c 2}$. In this case, the system undergoes two first-order quantum phase transitions: from the 111 state to a pseudospin density wave at $d_{c 1}$, and from the latter to two weakly coupled composite fermion Fermi liquids at $d_{c 2}$. The pseudospin density wave phase proposal is based on an earlier random phase approximation calculation, ${ }^{12}$ which finds that the (neutral) excitation spectrum has a minimum (magnetoroton) at finite momentum $\ell q \sim 1.0$ and that the energy of this mode vanishes at $d=$ $1.2 \ell$. This feature indicates that a phase that spontaneously breaks translational symmetry may be realized. Such an inhomogeneous phase is studied, for instance, in Ref. 17 within the Hartree-Fock approximation. Interestingly, it is shown that the ground-state energy is almost $d / \ell$ independent, similar to Fig. 4. However, we should recall that the finite-momentum $\mathrm{BEC}$ is indeed a homogeneous phase (see Sec. V).

\section{B. Consequences for the bilayer QHS at $\boldsymbol{v}_{T}=1$}

As discussed in Sec. III, the two approximation schemes used throughout this paper, the one- and two-mode approximations, impose some strong restrictions on the description of the finite-momentum BEC of magnetic excitons, but they allow us to carry out detailed calculations in order to verify the stability of such a phase. Therefore, they should be seen as an initial approach to study the finite-momentum BEC. A more elaborated approximation scheme is needed. Since the two-mode approximation suggests that the system reduces its energy by splitting the condensate into two equal pieces, a better approximation for the ground state should be a finitemomentum condensate such that all modes $\pm \mathbf{Q}_{i}$ with $Q_{i}=Q$ and $i=1, \ldots n$ are (equally) macroscopically occupied (see, e.g., Ref. 38 for the case $n \rightarrow \infty$ ). In particular, if $n \rightarrow \infty$, the cylindrical symmetry of the quasiparticle dispersion relation $\bar{\Omega}_{\mathbf{q}}$ would be restored. The implementation of such a scheme is rather involved and it will be deferred to a future publication.

However, the results that we have derived so far allow us to make the following statements about the bilayer QHS: (a) There are strong indications that a finite-momentum BEC phase is the most stable in the intermediate $d / \ell$ region. Such a state bears a strong similarity with the pseudospin spiral phase proposed by Park. ${ }^{21}$ (b) The instability of the zero-momentum BEC at $d \sim 0.5 \ell$, which we arrive at in Ref. 14, indeed corresponds to a first-order quantum phase transition from a zero-momentum BEC to a finite-momentum one. In principle, such a transition could be experimentally observed.

It is also worth mentioning that (c) according to the two-mode approximation, a finite-momentum $\mathrm{BEC}$ with $\ell Q=$ 1 is the lowest-energy configuration only for $d<1.6 \ell$, a value quite close to the critical $d_{c}$ where the incompressiblecompressible quantum phase transition is experimentally observed. Curiously, the Fermi momentum $\mathbf{k}_{F}$ of a composite fermion Fermi liquid at $v=1 / 2$ is $\ell k_{F}=1 .{ }^{10}$ The fact that $\ell Q>\ell k_{F}$ for $d>1.6 \ell$ could be an indication of the incompressible-compressible phase transition. However, we should emphasize that this is just an interesting observation since, at the moment, it is not possible to identify a composite fermion Fermi-liquid phase within our bosonization formalism.

\section{Open questions and next steps}

There are still a couple of open questions that we hope will be answered once the approximation discussed in the first paragraph of the previous section is carried out. It remains to be verified whether (i) the features found within the one- and two-mode approximations [points (a) and (b) 
above] are robust; (ii) the momentum $\ell Q_{0}$ associated with the lowest-energy configuration for a given $d / \ell$ (Fig. 8) either varies continuously with $d / \ell$ or is quantized $\ell Q_{0}=0,1,2, \ldots$; (iii) it is possible to identify a finite-momentum BEC with $\ell Q>1$ with two composite fermion Fermi liquids and/or with the pairing states proposed by Möller and co-workers. ${ }^{16}$ This would be an important step towards the determination of the nature of the incompressible-compressible phase transition.

In addition to studying a finite-momentum BEC where all modes $\mathbf{Q}_{i}$ with $\ell Q_{i}=\ell Q$ are macroscopically occupied, we also intend to consider the effects of a (small) finite electron interlayer tunneling, disorder (hopefully), and the electronic spin. Concerning the latter, there are some experimental evidences $^{39-41}$ that the electron spin degree of freedom might be relevant for a complete description of the bilayer QHS. For instance, it was recently reported ${ }^{40}$ that the critical $d_{c}$, where the incompressible-compressible phase transition takes place, increases and eventually saturates due to an increasing in-plane magnetic field $\mathbf{B}_{\|}$. In principle, the effects related to the electronic spin could be included in our analysis with the help of the generalized bosonization formalism, ${ }^{42}$ which has been recently proposed by two of us to study the quantum Hall effect in graphene at $v=0$ and \pm 1 .

So far, we have focused on the elementary neutral excitations of the bilayer QHS. It remains to be checked how charged excitations are described within our bosonization approach. Such excitations are important when disorder effects are taken into account (see, e.g., Refs. 43 and 44 and the references therein). Two distinct cases should be considered: (a) Zero-momentum BEC of magnetic excitons. Such a phase can also be seen as an $X Y$ pseudospin ferromagnet. ${ }^{45}$ In this language, charged excitations correspond to topological (vortex) excitations called merons. There are four types of merons: with vorticity \pm 1 and electric charge $\pm e / 2$. For the single-layer QHS at $v=1$, it was shown ${ }^{13}$ that a topological excitation (skyrmion) can be described as a boson coherent state. Due to the similarities between the single-layer and the bilayer QHSs, we expect that merons could be seen as a coherent state of bosons as well. (b) Finite-momentum BEC of magnetic excitons. Here, the mapping into an $X Y$ pseudospin model no longer holds (see note 14 in Ref. 21) and, therefore, it is not yet clear whether charged excitations could also be described as a boson coherent state.

Finally, concerning experiments, it would be interesting, e.g., to calculate the interlayer tunneling current for the finite-momentum BEC phase. The first theoretical works ${ }^{46}$ (clean limit) indicated that the bilayer QHS should display a Josephson-like effect, i.e., a zero-bias infinite tunneling conductance should be observed. Such a feature is related to the gapless linearly dispersing (neutral) excitation spectrum at low energies associated with the Halperin 111 phase (zeromomentum BEC of magnetic excitons). Instead, an enhanced finite tunneling conductance at zero-bias voltage was experimentally observed. ${ }^{4,5}$ In order to account for the experimental features, disorder effects were then considered. At the moment, the experimental data have been understood within an $X Y$ pseudospin model with the tunneling term (2) perturbatively treated and with disorder-induced merons phenomenologically included in the electron tunneling operator (for a review, see, e.g., Ref. 44 and the references therein). Interestingly, such a scheme indicates that by adding a parallel magnetic field $\mathbf{B}_{\|}$to the sample, the tunneling conductance versus bias voltage data could provide a measurement of the gapless linearly dispersing excitation spectrum. Again, the experimental data of Spielman et al., ${ }^{5}$ who found some evidences for the existence of such collective excitation, were analyzed within the above $X Y$ pseudospin framework.

In principle, our results are in contradiction with the experimental data ${ }^{5}$ since we have found a gapped phase in the intermediate $d / \ell$ region, where tunneling experiments were performed. However, note that according to our results, a true Josephson-like effect should occur only at very small $d / \ell$, where the zero-momentum BEC phase sets in. This is somehow in agreement with the experiments. Our next task is to verify whether the gapped excitation spectrum, Figs. 6 and 10 , could account for the observed finite tunneling conductance at zero-bias voltage. In principle, we can calculate the interlayer tunneling current (clean limit) within our bosonization formalism, treating the tunneling term (2) nonperturbatively. Disorder effects could be included later, for instance, following the lines of Ref. 43. In this way, we hope we can provide an alternative interpretation for the experimental data reported in Refs. 4 and 5.

\section{SUMMARY}

In this paper, we studied the bilayer QHS at $v_{T}=1$ within the bosonization method, ${ }^{13}$ a formalism which allows us to properly treat the magnetic exciton as a boson, and we showed that the ground state of the system in the region $d \sim \ell$ (zero interlayer tunneling case) can be seen as a finite-momentum BEC of magnetic excitons. Our findings are in agreement with previous results which suggest that an intermediate phase may show up between the (incompressible) Halperin 111 state (ground state for small $d / \ell$ ) and the (compressible) composite fermion Fermi liquids (ground state for larger $d / \ell$ ).

The stability of the finite-momentum BEC has been analyzed via two distinct approximation schemes: the onemode approximation, where it is assumed that the bosons macroscopically occupied only one momentum $\mathbf{Q}=Q \hat{x}$ with $\ell Q \neq 0$, and the two-mode approximation, where both $\pm \mathbf{Q}$ modes with $\mathbf{Q}=Q \hat{x}$ are macroscopically occupied. We have found that such a phase can be realized as long as the excitation spectrum is gapped. The comparison between the ground-state energy curves in terms of the ratio $d / \ell$ for configurations with different $\ell Q$ as well as the analysis of the quasiparticle excitation spectra provide strong evidences for a first-order quantum phase transition at small $d / \ell$, i.e., a transition from a zero-momentum BEC, a phase that we had already analyzed in Ref. 14 and that corresponds to Halperin 111 state, to a finite-momentum BEC. We hope that such first-order quantum phase transition can be experimentally observed in the near future.

As a final remark, we would like to emphasize that the bosonization method introduced in Ref. 13 can be used to study both the single- and double-layer QHSs at $v=1$. In other words, we can describe both systems using the same degree of freedom, the magnetic exciton, in the limit where this object can be treated as a boson. 


\section{ACKNOWLEDGMENTS}

We thank M. Vojta, L. Fritz, and A. Lazarides for helpful discussions. R.L.D. kindly acknowledges FAPESP (Project No. 10/00479-6) for the financial support; A.O.C. acknowledges the partial financial support from CNPq (Project No. 303073/2010-1) and FAPESP (Project No. 07/57630-5); and C.M.S. acknowledges the Netherlands Organization for Scientific Research (NWO).

\section{APPENDIX A: ABOUT THE BOSONIZATION SCHEME}

In Ref. 13, it is shown that a bosonization formalism for the two-dimensional electron gas under a strong magnetic field (single-layer QHS at $v=1$ ) can be developed following the lines of the bosonization scheme used to describe onedimensional electronic systems. It is interesting that this formalism also gives quite reasonable results for the bilayer QHS at $v_{T}=1$ even though such a system is in a limit very far from the one considered in Ref. 13. In this section, we start providing some heuristic arguments, which tell us why the bosonization scheme ${ }^{13}$ can also be employed to study the bilayer QHS at $\nu_{T}=1$, and later, we show a simple calculation which corroborates such arguments.

The bosonization method for the single-layer QHS at $v=$ 1 is based on the following points: The ground state of the (noninteracting) two-dimensional electron gas at $v=1$ is the quantum Hall ferromagnet $|\mathrm{FM}\rangle$ [Fig. 2(a)], the reference state. Elementary (neutral) excitations are electron-hole pairs or spin flips [Fig. 2(b)], which can be obtained by applying the spindensity operator $S^{-}$into $|\mathrm{FM}\rangle$, i.e., $|\Psi\rangle \propto S^{-}|\mathrm{FM}\rangle$. Although the commutation relation between the spin-density operators $S^{+}$and $S^{-}$(projected into the lowest Landau level),

$$
\left[S_{\mathbf{q}}^{+}, S_{\mathbf{k}}^{-}\right]=e^{l^{2} q k^{*} / 2} \rho_{\uparrow}(\mathbf{q}+\mathbf{k})-e^{l^{2} k q^{*} / 2} \rho_{\downarrow}(\mathbf{q}+\mathbf{k})
$$

with $q=q_{x}+i q_{y}$, differs from the canonical commutation relation between creation and annihilation boson operators, it is still possible to define boson operators $b$ as in Eq. (9) if we follow the lines of Tomonaga's procedure ${ }^{33}$ for onedimensional electron systems. Using the fact that

$$
\begin{aligned}
& \rho_{\uparrow}(\mathbf{q}) \approx\left\langle\mathrm{FM}\left|\rho_{\uparrow}(\mathbf{q})\right| \mathrm{FM}\right\rangle=N_{\phi} \delta_{\mathbf{q}, 0}, \\
& \rho_{\downarrow}(\mathbf{q}) \approx\left\langle\mathrm{FM}\left|\rho_{\downarrow}(\mathbf{q})\right| \mathrm{FM}\right\rangle=0,
\end{aligned}
$$

we realize that Eq. (A1) assumes the form

$$
\left[S_{\mathbf{q}}^{+}, S_{\mathbf{k}}^{-}\right] \approx N_{\phi} \exp \left[-(l q)^{2} / 2\right] \delta_{\mathbf{q}+\mathbf{k}, 0},
$$

which now resembles a canonical commutation relation for boson operators. In other words, as long as we are close to the $|\mathrm{FM}\rangle$ state, i.e., the number of bosons in the system is small, electron-hole excitations (magnetic excitons) can be approximately treated as bosons.

Turning to the bilayer QHS at $\nu_{T}=1$, we note that such a system is very far from the limit discussed above because the configuration $v_{T}=v_{\uparrow}+v_{\downarrow}=1 / 2+1 / 2=1$ corresponds to a system with $N_{\phi} / 2$ bosons [see Fig. 1(b)]. As we will see below, this is indeed a very special configuration where density fluctuations guarantee that the relation (A3) still holds.

Let us consider as a reference state the zero-momentum BEC of magnetic excitons illustrated in Fig. 1(b) and identify the electronic spin degree of freedom of the single layer with the pseudospin one of the bilayer QHS. In this case, we have

$$
\begin{aligned}
& \rho_{\uparrow}(\mathbf{q}) \approx\left\langle\rho_{\uparrow}(\mathbf{q})\right\rangle+\delta \rho_{\uparrow}(\mathbf{q}), \\
& \rho_{\downarrow}(\mathbf{q}) \approx\left\langle\rho_{\downarrow}(\mathbf{q})\right\rangle+\delta \rho_{\downarrow}(\mathbf{q}) .
\end{aligned}
$$

Since $\left\langle\rho_{\uparrow}(\mathbf{q})\right\rangle=\left\langle\rho_{\downarrow}(\mathbf{q})\right\rangle=\left(N_{\phi} / 2\right) \delta_{\mathbf{q}, 0}$, we note that here the equivalent of Eq. (A3) vanishes, and therefore the bosons $b$ are no longer well defined. However, since the total filling factor is fixed, we have

$$
\delta \rho_{\uparrow}(\mathbf{q})=-\delta \rho_{\downarrow}(\mathbf{q})=\delta \rho(\mathbf{q}),
$$

which indicates that a finite (approximate) commutation relation can still be obtained if we now consider density fluctuations in Eq. (A4). Due to the relation between the magnetic exciton momentum $\mathbf{q}$ and the guiding centers associated with the electron and the hole, we have

$$
\delta \rho(\mathbf{q})=\delta \rho_{\text {local }}(\mathbf{q})+\delta \rho_{\text {nonlocal }}(\mathbf{q}),
$$

where $\delta \rho_{\text {local }}(\mathbf{q})$ and $\delta \rho_{\text {nonlocal }}(\mathbf{q})$ correspond to density fluctuations on the same and different guiding centers, respectively. Moreover, we can also write

$$
\delta \rho_{\text {local }}(\mathbf{q}) \approx \rho_{0} \delta_{\mathbf{q}, 0},
$$

with $\rho_{0}$ being a constant, which implies that the relation (A3) still holds for the bilayer QHS at $v_{T}=1$.

In order to see that (A6) and (A7) are indeed quite reasonable assumptions, let us calculate the density fluctuations

$$
\delta \rho_{\alpha}(\mathbf{q})=\sqrt{\left\langle\rho_{\alpha}^{2}(\mathbf{q})\right\rangle-\left\langle\rho_{\alpha}(\mathbf{q})\right\rangle^{2}}
$$

within the two-mode approximation. It can be seen as a kind of self-consistent check of the above assumptions.

In the Bogoliubov approximation, the Fourier transform of the $\alpha$-electron density operator reads as [see Eqs. (25) and (26) from Ref. 13]

$$
\begin{aligned}
\rho_{\alpha}(\mathbf{q}) & =\delta_{\alpha, \uparrow} \delta_{q, 0} N_{\Phi}-\alpha e^{-(\ell q)^{2} / 4}\left[e^{-i \alpha \mathbf{q} \wedge \mathbf{Q} / 2} \sqrt{N_{0}} b_{\mathbf{Q}+\mathbf{q}}^{\dagger}\right. \\
& +e^{i \alpha \mathbf{q} \wedge \mathbf{Q} / 2 \sqrt{\bar{N}_{0}} b_{-\mathbf{Q}+\mathbf{q}}^{\dagger}} \\
& \left.+\sum_{\mathbf{k} \neq 0,-2 \mathbf{Q}} e^{-i \alpha \mathbf{q} \wedge(\mathbf{Q}+\mathbf{k}) / 2} b_{\mathbf{Q}+\mathbf{k}+\mathbf{q}}^{\dagger} b_{\mathbf{Q}+\mathbf{k}}\right]
\end{aligned}
$$

Since

$$
\begin{aligned}
\left\langle b_{\mathbf{Q}+\mathbf{q}}^{\dagger}\right\rangle & =\delta_{\mathbf{q}, 0} N_{0}^{1 / 2}+\delta_{\mathbf{q},-2 \mathbf{Q}} \bar{N}_{0}^{1 / 2}, \\
\left\langle b_{-\mathbf{Q}+\mathbf{q}}^{\dagger}\right\rangle & =\delta_{\mathbf{q}, 0} \bar{N}_{0}^{1 / 2}+\delta_{\mathbf{q}, 2 \mathbf{Q}} N_{0}^{1 / 2},
\end{aligned}
$$

and

$$
\begin{aligned}
\left\langle b_{\mathbf{Q}+\mathbf{k}+\mathbf{q}}^{\dagger} b_{\mathbf{Q}+\mathbf{k}}\right\rangle= & \delta_{\mathbf{q}, 0}\left\langle b_{\mathbf{Q}+\mathbf{k}}^{\dagger} b_{\mathbf{Q}+\mathbf{k}}\right\rangle+\delta_{\mathbf{q}, 2 \mathbf{Q}}\left\langle b_{3 \mathbf{Q}+\mathbf{k}}^{\dagger} b_{\mathbf{Q}+\mathbf{k}}\right\rangle \\
& +\delta_{\mathbf{q},-2 \mathbf{Q}}\left\langle b_{-\mathbf{Q}+\mathbf{k}}^{\dagger} b_{\mathbf{Q}+\mathbf{k}}\right\rangle,
\end{aligned}
$$

where the expectation value is taken with respect to the ground state of the bilayer QHS, i.e., the vacuum for the bosons $a$ [see Eq. (36)], it follows that

$$
\left\langle\rho_{\alpha}(\mathbf{q})\right\rangle=\delta_{\mathbf{q}, 0} \frac{1}{2} N_{\phi}-\left(\delta_{\mathbf{q}, 2 \mathbf{Q}}+\delta_{\mathbf{q},-2 \mathbf{Q}}\right) e^{-|\ell \mathbf{Q}|^{2}} F_{\alpha}(\mathbf{q}) .
$$


Here,

$$
\begin{aligned}
F_{\alpha}(\mathbf{q}) \equiv & \sqrt{N_{0} \bar{N}_{0}} \\
& +\sum_{\mathbf{k} \neq 0,2 \alpha \mathbf{Q}} e^{i \alpha \mathbf{q} \wedge \mathbf{k} / 2}\left[v_{1}(\mathbf{k}) v_{2}(\mathbf{k})+v_{3}(\mathbf{k}) v_{4}(\mathbf{k})\right],
\end{aligned}
$$

with $v_{i}(\mathbf{q})$ being the Bogoliubov coefficients [Eq. (C5)]. Concerning $\left\langle\rho_{\alpha}^{2}(\mathbf{q})\right\rangle$, after some algebra, it is possible to show that

$$
\begin{aligned}
\left\langle\rho_{\alpha}(\mathbf{q}) \rho_{\alpha}(\mathbf{q})\right\rangle= & \delta_{\mathbf{q}, 0}\left[\frac{1}{4} N_{\phi}^{2}+\rho_{0}\right]+\left[\delta_{\mathbf{q}, 2 \mathbf{Q}}+\delta_{\mathbf{q},-2 \mathbf{Q}}\right] \\
& \times e^{-2|\ell \mathbf{Q}|^{2}}\left[F_{\alpha}^{2}(\mathbf{q})+\rho_{1}(\mathbf{q})\right],
\end{aligned}
$$

where

$$
\begin{aligned}
\rho_{0}= & \sum_{\mathbf{p}}\left[v_{1}^{2}(\mathbf{p})+v_{3}^{2}(\mathbf{p})\right]^{2}+\left[u_{1}(\mathbf{p}) v_{2}(\mathbf{p})+u_{3}(\mathbf{p}) v_{4}(\mathbf{p})\right]^{2} \\
& +2\left[v_{1}(\mathbf{p}) v_{2}(\mathbf{p})+v_{3}(\mathbf{p}) v_{4}(\mathbf{p})\right]^{2} \\
& +2\left[u_{1}(\mathbf{p}) v_{1}(\mathbf{p})+u_{3}(\mathbf{p}) v_{3}(\mathbf{p})\right]^{2} \\
\rho_{1}(\mathbf{q})= & \sum_{\mathbf{p}} \cos (\mathbf{q} \wedge \mathbf{p})\left[v_{1}(\mathbf{p}) v_{2}(\mathbf{p})+v_{3}(\mathbf{p}) v_{4}(\mathbf{p})\right]^{2} \\
& +\left[u_{1}(\mathbf{p}) v_{2}(\mathbf{p})+u_{3}(\mathbf{p}) v_{4}(\mathbf{p})\right]^{2} .
\end{aligned}
$$

Therefore, Eqs. (A5), (A8), (A10), and (A11) yield

$$
\delta \rho^{2}(\mathbf{q})=\delta_{\mathbf{q}, 0} \rho_{0}+\left[\delta_{\mathbf{q}, 2 \mathbf{Q}}+\delta_{\mathbf{q},-2 \mathbf{Q}}\right] e^{-2|\ell \mathbf{Q}|^{2}} \rho_{1}(\mathbf{q}) .
$$

Note that the first term of the above equation can be identified with $\delta \rho_{\text {local }}(\mathbf{q})$ in (A6), while the second one with $\delta \rho_{\text {nonlocal }}(\mathbf{q})$. Moreover, one can easily see that $\rho_{0}>e^{-2|\ell \mathbf{Q}|^{2}} \rho_{1}( \pm \mathbf{Q})$, which corroborates the fact that $\delta \rho_{\text {nonlocal }}(\mathbf{q})$ can be neglected with respect to $\delta \rho_{\text {local }}(\mathbf{q})$.

In the one-mode approximation, Eqs. (A10) and (A11) reduce to

$$
\begin{aligned}
\left\langle\rho_{\alpha}(\mathbf{q})\right\rangle & =\delta_{\mathbf{q}, 0} \frac{1}{2} N_{\phi}, \\
\left\langle\rho_{\alpha}(\mathbf{q}) \rho_{\sigma}(\mathbf{q})\right\rangle & =\delta_{\mathbf{q}, 0}\left[\frac{1}{4} N_{\phi}^{2}+\frac{1}{2} \sum_{\mathbf{k} \neq \mathbf{Q}} \frac{\epsilon_{\mathbf{k}}\left(\epsilon_{\mathbf{k}}-\Omega_{\mathbf{k}}\right)}{\Omega_{\mathbf{k}}^{2}}\right] .
\end{aligned}
$$

Although $\delta \rho_{\text {nonlocal }}(\mathbf{q})=0$, the bosonization scheme ${ }^{13}$ still holds because the relevant term $\delta \rho_{\text {local }}(\mathbf{q})$ is finite within this approximation.

\section{APPENDIX B: ALTERNATIVE EFFECTIVE BOSON MODELS FOR THE BILAYER QHS}

In this section, we briefly comment on some different effective boson models proposed to describe the bilayer QHS.

In Ref. 47, the effects of the electron spin degree of freedom are taken into account. Here, the original fermion model is mapped into an effective lattice spin-pseudospin model, which is then analyzed within a generalized Schwinger boson meanfield theory. Finite-temperature properties are calculated, for instance, the temperature dependence of the spin and in-plane pseudospin magnetizations. A proper comparison between our results and those of Ref. 47 will be possible only after including the electronic spin in our formalism (see Sec. VIC). We would like to recall that our approach is based on a direct mapping of the interacting fermion model (1) into the boson model (12), no lattice degrees of freedom are introduced.

Tieleman and co-workers ${ }^{48}$ derived an effective boson model for the bilayer QHS also following the ideas of the bosonization scheme,${ }^{13}$ but with some important differences: The single-particle electron states considered are no longer the pseudospin-up and -down lowest Landau levels (see Fig. 1), but symmetric and antisymmetric linear combination of these states. Instead of defining the boson operators with respect to the quantum Hall ferromagnet $|\mathrm{FM}\rangle$ (see Sec. II A), the reference state is the completely filled symmetric state $|\mathrm{SYM}\rangle$. Therefore, the boson operators introduced in Ref. 48, hereafter called $\beta$, differ from the bosons $b$ discussed in Sec. II A. Most importantly, the ground state corresponds to an almost filled symmetric state instead of the BEC of magnetic excitons considered within our formalism. Since the procedure adopted in Ref. 48 to derive an effective boson model is not fully consistent with the bosonization scheme,${ }^{13}$ in the following we briefly revisit its derivation.

Formally, the bosonization schemes of Refs. 13 and 48 are identical. Therefore, the bosonic representations of the electron density and pseudospin density operators are given by Eqs. (27)-(29) and (31) of Ref. 13 with the replacement $b \rightarrow \beta$. Substituting these expressions in Eq. (7) of Ref. 48, we arrive at

$$
H_{B}^{\mathrm{SAS}}=\mathrm{H}_{2}+\mathrm{H}_{4}+H_{6},
$$

where $H_{2,4,6}$ respectively correspond to terms with 2,4 , and 6 boson operators $\beta$. In particular,

$$
H_{2}=\sum_{\mathbf{q}} \tilde{\epsilon}_{\mathbf{q}} \beta_{\mathbf{q}}^{\dagger} \beta_{\mathbf{q}}+\frac{1}{2}\left(\tilde{\lambda}_{\mathbf{q}} \beta_{\mathbf{q}}^{\dagger} \beta_{-\mathbf{q}}^{\dagger}+\bar{\lambda}_{\mathbf{q}} \beta_{-\mathbf{q}} \beta_{\mathbf{q}}\right),
$$

with

$$
\begin{aligned}
\tilde{\epsilon}_{\mathbf{q}}= & t+\tilde{\lambda}_{\mathbf{q}}-\sum_{\mathbf{k}} e^{-(\ell k)^{2} / 2} v_{c}(k) \\
& +\sum_{\mathbf{k}} 2 v_{0}(k) e^{-(\ell k)^{2} / 2} \sin ^{2}(\mathbf{k} \wedge \mathbf{q} / 2), \\
\tilde{\lambda}_{\mathbf{q}}= & N_{\phi} e^{-(\ell p)^{2} / 2} v_{c}(q), \\
\bar{\lambda}_{\mathbf{q}}= & \tilde{\lambda}_{\mathbf{q}}-\sum_{\mathbf{k}} v_{c}(k) e^{-(l k)^{2} / 2} \cos (\mathbf{k} \wedge \mathbf{q}) .
\end{aligned}
$$

Here, $t$ and $v_{0 / c}(k)$, respectively, correspond to $\Delta_{\mathrm{SAS}}$ and $v_{0 / z}(k)$ (see Sec. II). Comparing Eqs. (B1)-(B3) with equations (19)-(21) of Ref. 48, one can see that the former have extra terms. This is related to the fact that Tieleman et al. approximated the bosonic representation of the operator $S_{x}(\mathbf{k})$ by linear terms, i.e., $S_{x}(\mathbf{k}) \sim \beta_{\mathbf{k}}^{\dagger}+\beta_{-\mathbf{k}}$, while here the complete bosonic representation, which in addition includes a cubic term in boson operators $\beta$, is considered. We should mention that the presence of such a cubic term in the bosonic representation of $S_{x}(\mathbf{k})$ is important because it guarantees that the bosonic representation of the electron density and spindensity operators obey the correct commutation relations, the so-called lowest Landau level algebra (see Sec. II D of Ref. 13 for details). In order to perform a proper map of the original fermion model into the boson one, we should consider the complete bosonic expression of the electron density and spindensity operators. After that, approximations can be employed. 
Therefore, if we define boson operators with respect to the state $|\mathrm{SYM}\rangle$ and try to be consistent with the bosonization formalism, ${ }^{13}$ we then obtain a non-Hermitian effective boson model to describe the bilayer QHS. Recall that the procedure adopted in this paper yields a Hermitian boson model [Eq. (12)].

Although the Hamiltonian (B1) is non-Hermitian, let us for the moment assume that this is only an artifact of the bosonization scheme and determine the spectrum of the elementary excitations $\Omega_{\mathbf{q}}$ within the harmonic approximation,

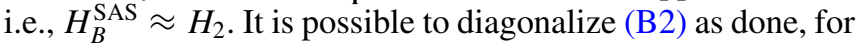
instance, in Ref. 49. One finds

$$
\Omega_{\mathbf{q}}=\sqrt{\tilde{\epsilon}_{\mathbf{q}}^{2}-\tilde{\lambda}_{\mathbf{q}} \bar{\lambda}_{\mathbf{q}}}
$$

However, $\Omega_{\mathbf{q}}$ is not well defined for small momenta: it is easy to see that $\tilde{\epsilon}_{\mathbf{q}}^{2}-\tilde{\lambda}_{\mathbf{q}} \bar{\lambda}_{\mathbf{q}}<0$ for $q \rightarrow 0$. One concludes that the restriction of $H_{B}^{\mathrm{SAS}}$ to the quadratic term (B2) is not a good approximation. A proper analysis of $H_{B}^{\mathrm{SAS}}$ should take into account the $H_{4}$ and $H_{6}$ terms. Finally, one should mention that the approach of Ref. 48 is more suitable for larger $\Delta_{\text {SAS }}$ values, while our formalism is for the opposite limit.

Interestingly, apart from the coefficient of the $\beta_{\mathbf{q}}^{\dagger} \beta_{-\mathbf{q}}^{\dagger}$ term, the Hamiltonian (B1) corresponds to the effective boson model proposed in Ref. 50: Here the energy of the elementary excitations is given by $\Omega_{\mathbf{q}}=\left(\tilde{\epsilon}_{\mathbf{q}}^{2}-\bar{\lambda}_{\mathbf{q}}^{2}\right)^{1 / 2}$, which agrees with the diagrammatic calculations of Fertig. ${ }^{12}$ Recall that, in this case, the spectrum has a magnetoroton minimum which vanishes for $d \approx 1.2 \ell$ ( $t=0$ case $)$.

\section{APPENDIX C: DETAILS: TWO-MODE APPROXIMATION}

In this Appendix, we provide the full expressions of some quantities which appear in Sec. IV.

After the substitution (27), Eq. (15) acquires the form

$$
\begin{aligned}
K= & K_{0}+\frac{1}{4} \sum_{\mathbf{q}}^{\prime}\left[\epsilon_{\mathbf{q}}^{+}\left(b_{\mathbf{Q}+\mathbf{q}}^{\dagger} b_{\mathbf{Q}+\mathbf{q}}+b_{-\mathbf{Q}-\mathbf{q}} b_{-\mathbf{Q}-\mathbf{q}}^{\dagger}\right)\right. \\
& +\epsilon_{\mathbf{q}}^{-}\left(b_{\mathbf{Q}-\mathbf{q}} b_{\mathbf{Q}-\mathbf{q}}^{\dagger}+b_{-\mathbf{Q}+\mathbf{q}}^{\dagger} b_{-\mathbf{Q}+\mathbf{q}}\right) \\
& +\gamma_{\mathbf{q}}\left(b_{\mathbf{Q}+\mathbf{q}}^{\dagger} b_{-\mathbf{Q}+\mathbf{q}}+\text { H.c. }+b_{-\mathbf{Q}-\mathbf{q}}^{\dagger} b_{\mathbf{Q}-\mathbf{q}}+\text { H.c. }\right) \\
& +\lambda_{\mathbf{q}}\left(b_{\mathbf{Q}+\mathbf{q}}^{\dagger} b_{\mathbf{Q}-\mathbf{q}}^{\dagger}+\text { H.c. }\right)+\bar{\lambda}_{\mathbf{q}}\left(b_{-\mathbf{Q}+\mathbf{q}}^{\dagger} b_{-\mathbf{Q}-\mathbf{q}}^{\dagger}+\text { H.c. }\right) \\
& \left.+\xi_{\mathbf{q}}\left(b_{-\mathbf{Q}+\mathbf{q}}^{\dagger} b_{\mathbf{Q}-\mathbf{q}}^{\dagger}+\text { H.c. }+b_{\mathbf{Q}+\mathbf{q}}^{\dagger} b_{-\mathbf{Q}-\mathbf{q}}^{\dagger}+\text { H.c. }\right)\right] .
\end{aligned}
$$

The restriction on the sum over momenta indicates that the modes that satisfy the condition $\pm \mathbf{Q} \pm \mathbf{q} \neq \pm \mathbf{Q}$ are not included. Here,

$$
\begin{aligned}
K_{0} & =2 N_{0} \bar{N}_{0} v_{2 \mathbf{Q}}(\mathbf{Q}, \mathbf{Q})+\left(\omega_{\mathbf{Q}}-\mu\right)\left(N_{0}+\bar{N}_{0}\right)-\frac{1}{2} \sum_{\mathbf{q}} \epsilon_{\mathbf{q}}, \\
\gamma_{\mathbf{q}} & =4 \sqrt{N_{0} \bar{N}_{0}}\left[v_{\mathbf{q}}(-\mathbf{Q}, \mathbf{Q})+v_{2 \mathbf{Q}}(\mathbf{q}, \mathbf{Q})\right], \\
\lambda_{\mathbf{q}} & =4 N_{0} v_{\mathbf{q}}(\mathbf{Q}, \mathbf{Q}), \quad \bar{\lambda}_{\mathbf{q}}=4 \bar{N}_{0} v_{\mathbf{q}}(\mathbf{Q}, \mathbf{Q}), \\
\xi_{\mathbf{q}} & =4 \sqrt{N_{0} \bar{N}_{0}} v_{\mathbf{q}}(-\mathbf{Q}, \mathbf{Q}),
\end{aligned}
$$

and $\epsilon_{\mathbf{q}}^{ \pm}$are defined in Eq. (18). Setting $\bar{N}_{0}=N_{0}$ and using the fact that $n_{0}=N_{0} / N_{B}=4 \pi \ell^{2} N_{0}$, we can write

$$
\begin{aligned}
& \lambda_{\mathbf{q}}=\frac{e^{2}}{\epsilon \ell}\left(2 n_{0}\right) \frac{e^{-(\ell q)^{2} / 2}}{\ell q}\left[1-e^{-(\ell q) d / \ell} \cos (\mathbf{q} \wedge \mathbf{Q})\right], \\
& \xi_{\mathbf{q}}=\frac{e^{2}}{\epsilon \ell}\left(2 n_{0}\right) \frac{e^{-(\ell q)^{2} / 2}}{\ell q}\left[\cos (\mathbf{q} \wedge \mathbf{Q})-e^{-(\ell q) d / \ell}\right], \\
& \gamma_{\mathbf{q}}=\xi_{\mathbf{q}}+\frac{e^{2}}{\epsilon \ell} n_{0} \frac{e^{-(\ell q)^{2} / 2}}{\ell q}\left[1-e^{-2(\ell q) d / \ell}\right] \cos (\mathbf{q} \wedge \mathbf{Q}),
\end{aligned}
$$

which are useful expressions for the numerical calculations.

Concerning the chemical potential Eq. (41), the terms $\mu_{0}$ and $\mu_{1}$ are defined as

$$
\begin{aligned}
\mu_{0}= & \frac{1}{8 N_{0}} \sum_{\mathbf{q}}\left(\frac{1}{\Omega_{\mathbf{q}}^{+}}+\frac{1}{\Omega_{\mathbf{q}}^{-}}\right)\left[\lambda_{\mathbf{q}}\left(\epsilon_{\mathbf{q}}-\lambda_{\mathbf{q}}\right)+\gamma_{\mathbf{q}}^{2}-\xi_{\mathbf{q}}^{2}\right] \\
& -2 \lambda_{\mathbf{q}}+\frac{1}{D_{\mathbf{q}}}\left(\frac{1}{\Omega_{\mathbf{q}}^{+}}-\frac{1}{\Omega_{\mathbf{q}}^{-}}\right)\left[\Delta_{\mathbf{q}}^{2} \lambda_{\mathbf{q}}\left(\epsilon_{\mathbf{q}}-\lambda_{\mathbf{q}}\right)\right. \\
& \left.+\left(\gamma_{\mathbf{q}} \epsilon_{\mathbf{q}}-\gamma_{\mathbf{q}} \xi_{\mathbf{q}}\right)\left(\gamma_{\mathbf{q}} \epsilon_{\mathbf{q}}+\gamma_{\mathbf{q}} \lambda_{\mathbf{q}}-2 \lambda_{\mathbf{q}} \xi_{\mathbf{q}}\right)\right], \\
\mu_{1}= & 2 N_{0} v_{2 \mathbf{Q}}(\mathbf{Q}, \mathbf{Q})=\frac{e^{2}}{\epsilon \ell} \frac{1}{2} n_{0} \frac{e^{-2(\ell Q)^{2}}}{\ell Q}\left[1-e^{-2(\ell Q) d / \ell}\right] .
\end{aligned}
$$

Finally, following the procedure described in Ref. 31 (see Chap. 3) and after some algebra, it is possible to show that the Bogoliubov coefficients $u_{i}(\mathbf{q})$ and $v_{i}(\mathbf{q})$, the elements of the $4 \times 4$ matrix $\hat{M}_{\mathbf{q}}$ [Eq. (37)] are given by

$$
\begin{aligned}
u_{i}^{2}(\mathbf{q}), v_{i}^{2}(\mathbf{q})= & \frac{1}{4 \Omega_{\mathbf{q}}^{i}}\left(\epsilon_{\mathbf{q}}+\alpha_{i} \Delta_{\mathbf{q}} \pm \Omega_{\mathbf{q}}^{i}\right) \\
& -\frac{(-1)^{i}}{4 \Omega_{\mathbf{q}}^{i} D_{\mathbf{q}}}\left[\gamma_{\mathbf{q}}\left(\gamma_{\mathbf{q}} \epsilon_{\mathbf{q}}-\lambda_{\mathbf{q}} \xi_{\mathbf{q}}\right)-\alpha_{i} \Delta_{\mathbf{q}} \lambda_{\mathbf{q}}^{2}\right. \\
& \left.+\Delta_{\mathbf{q}} \epsilon_{\mathbf{q}}\left(\alpha_{i} \epsilon_{\mathbf{q}}+\Delta_{\mathbf{q}} \pm \alpha_{i} \Omega_{\mathbf{q}}^{i}\right)\right]
\end{aligned}
$$

with $i=1,2,3,4, \Omega_{\mathbf{q}}^{i}=\Omega_{\mathbf{q}}^{+}(i=1,3), \Omega_{\mathbf{q}}^{-}(i=2,4)$, and $\alpha_{i}=1(i=1,2),-1(i=3,4)$.

\section{APPENDIX D: PAIR CORRELATION FUNCTION}

In this section, we present the full expressions for the pair correlation function (46), calculated both at the one- and twomode approximations. In order to calculate the static structure factor (47), it is necessary to project the product $\rho(-\mathbf{q}) \rho(\mathbf{q})$ into the lowest Landau level. We have [see Eqs. (4.17) and (4.18) from Ref. 36]

$$
\overline{\rho(-\mathbf{q}) \rho(\mathbf{q})}=\bar{\rho}(-\mathbf{q}) \bar{\rho}(\mathbf{q})+N\left[1-\exp \left(-|\ell \mathbf{q}|^{2} / 2\right)\right],
$$

and therefore Eq. (47) assumes the form

$$
S(\mathbf{q})=\bar{S}(\mathbf{q})+\left[1-\exp \left(-|\ell \mathbf{q}|^{2} / 2\right)\right]
$$

with $\bar{S}(\mathbf{q})=(1 / N)\langle\bar{\rho}(-\mathbf{q}) \bar{\rho}(\mathbf{q})\rangle-N \delta_{\mathbf{q}, 0}$ being the projected static structure factor. From Eq. (10), it follows that $(\mathbf{q} \neq 0)$

$$
\begin{aligned}
\bar{S}(\mathbf{q})= & \frac{4 e^{-|\ell q|^{2} / 2}}{N_{\phi}} \sum_{\mathbf{p}, \mathbf{k}} \sin (\mathbf{q} \wedge \mathbf{p} / 2) \sin (\mathbf{q} \wedge \mathbf{k} / 2) \\
& \times\left\langle b_{\mathbf{k}-\mathbf{q}}^{\dagger} b_{\mathbf{k}} b_{\mathbf{p}+\mathbf{q}}^{\dagger} b_{\mathbf{p}}\right\rangle .
\end{aligned}
$$


Here, the $\exp \left(-|\ell q|^{2} / 4\right)$ factor is restored in the bosonic representation of the electron density operator [see Eq. (27) from Ref. 13].

Using Wick's theorem, the expectation value in the above equation can be easily calculated. Performing the replacement $b_{\mathbf{Q}}^{\dagger}, b_{\mathbf{Q}} \rightarrow \sqrt{N_{0}}$ as done in Sec. III and keeping only the terms with two boson operators $b$, it is possible to show that in the one-mode approximation the projected static structure factor assumes the form

$$
\bar{S}(\mathbf{q}) \approx 2 n_{0} e^{-|\ell|^{2} / 2} \sin ^{2}(\mathbf{q} \wedge \mathbf{Q} / 2)\left(v_{\mathbf{q}}^{2}+u_{\mathbf{q}}^{2}-2 u_{\mathbf{q}} v_{\mathbf{q}}\right)
$$

with the Bogoliubov coefficients $u_{\mathbf{q}}$ and $v_{\mathbf{q}}$ given by Eq. (23). Note that $\bar{S}(\mathbf{q})$ vanishes if $\mathbf{Q}=0$ implying that, within this level of approximation,

$$
g(r)=1-\exp \left[-r^{2} /\left(2 \ell^{2}\right)\right]
$$

regardless the value of $d / \ell$. Equation (D5) is nothing else but the pair correlation function for the single-layer QHS at $v=1$.

The same procedure can be used to calculate $g(r)$ in the two-mode approximation. After some algebra, we find that

$$
\begin{aligned}
\bar{S}(\mathbf{q}) \approx & 2 n_{0} e^{-|\ell q|^{2} / 2} \sin ^{2}(\mathbf{q} \wedge \mathbf{Q}) \\
& \times\left[\left[u_{1}(\mathbf{q})+v_{1}(\mathbf{q})-u_{2}(\mathbf{q})-v_{2}(\mathbf{q})\right]^{2}\right. \\
& \left.+\left[u_{3}(\mathbf{q})+v_{3}(\mathbf{q})-u_{4}(\mathbf{q})-v_{4}(\mathbf{q})\right]^{2}\right],
\end{aligned}
$$

where the Bogoliubov coefficients $u_{i}(\mathbf{q})$ and $v_{i}(\mathbf{q})$ are now given by Eq. (C5).
${ }^{1}$ For an introduction, see Perspectives in Quantum Hall Effects, edited by S. Das Sarma and A. Pinczuk (Wiley, New York, 1997); The Quantum Hall Effect, edited by D. Yoshioka (Springer, Berlin, 2002).

${ }^{2}$ J. P. Eisenstein and A. H. MacDonald, Nature (London) 432, 691 (2004); J. P. Eisenstein, Science 305, 950 (2004).

${ }^{3}$ S. Q. Murphy, J. P. Eisenstein, G. S. Boebinger, L. N. Pfeiffer, and K. W. West, Phys. Rev. Lett. 72, 728 (1994); M. Kellogg, I. B. Spielman, J. P. Eisenstein, L. N. Pfeiffer, and K. W. West, ibid. 88, 126804 (2002); M. Kellogg, J. P. Eisenstein, L. N. Pfeiffer, and K. W. West, ibid. 93, 036801 (2004); Y. Yoon, L. Tiemann, S. Schmult, W. Dietsche, K. von Klitzing, and W. Wegscheider, ibid. 104, 116802 (2010).

${ }^{4}$ I. B. Spielman, J. P. Eisenstein, L. N. Pfeiffer, and K. W. West, Phys. Rev. Lett. 84, 5808 (2000).

${ }^{5}$ I. B. Spielman, J. P. Eisenstein, L. N. Pfeiffer, and K. W. West, Phys. Rev. Lett. 87, 036803 (2001).

${ }^{6}$ G. Möller, S. H. Simon, and E. H. Rezayi, Phys. Rev. B 79, 125106 (2009).

${ }^{7}$ J. Schliemann, S. M. Girvin, and A. H. MacDonald, Phys. Rev. Lett. 86, 1849 (2001).

${ }^{8}$ J. Ye and L. Jiang, Phys. Rev. Lett. 98, 236802 (2007).

${ }^{9}$ B. I. Halperin, Helv. Phys. Acta 56, 75 (1983).

${ }^{10}$ B. I. Halperin, Patrick A. Lee, and N. Read, Phys. Rev. B 47, 7312 (1993).

${ }^{11}$ Composite Fermions, edited by O. Heinonen (World Scientific, Singapore, 1998).

${ }^{12}$ H. A. Fertig, Phys. Rev. B 40, 1087 (1989).

${ }^{13}$ R. L. Doretto, A. O. Caldeira, and S. M. Girvin, Phys. Rev. B 71, 045339 (2005).

${ }^{14}$ R. L. Doretto, A. O. Caldeira, and C. M. Smith, Phys. Rev. Lett. 97, 186401 (2006).

${ }^{15}$ S. H. Simon, E. H. Rezayi, and M. V. Milovanovic, Phys. Rev. Lett. 91, 046803 (2003).

${ }^{16}$ G. Möller, S. H. Simon, and E. H. Rezayi, Phys. Rev. Lett. 101, 176803 (2008).

${ }^{17}$ R. Côté, L. Brey, and A. H. MacDonald, Phys. Rev. B 46, 10239 (1992).

${ }^{18}$ Y. B. Kim, C. Nayak, E. Demler, N. Read, and S. Das Sarma, Phys. Rev. B 63, 205315 (2001).
${ }^{19}$ K. Nomura and D. Yoshioka, Phys. Rev. B 66, 153310 (2002).

${ }^{20}$ J. Schliemann, Phys. Rev. B 67, 035328 (2003).

${ }^{21}$ K. Park, Phys. Rev. B 69, 045319 (2004).

${ }^{22}$ M. V. Milovanović, Phys. Rev. B 75, 035314 (2007)

${ }^{23}$ The dispersion relation of the free bosons in Ref. 14 has an extra term $-\left(e^{2} / \epsilon \ell\right)(d / \ell)$. This contribution is related to the $\mathbf{k}=0$ term of the interacting potential (3) which was not properly removed in our first analysis. There are no modifications in the results obtained in Ref. 14 since they all depend on the difference $\omega_{\mathbf{q}}-\omega_{0}$.

${ }^{24}$ C. Kallin and B. I. Halperin, Phys. Rev. B 30, 5655 (1984).

${ }^{25}$ T. D. Stanescu, B. Anderson, and V. Galitski, Phys. Rev. A 78, 023616 (2008).

${ }^{26}$ M. DiLiberto, O. Tieleman, V. Branchina, and C. M. Smith, Phys. Rev. A 84, 013607 (2011).

${ }^{27}$ A. L. Fetter and J. D. Walecka, Quantum Theory of Many Particle Systems (Dover, Mineola, New York, 2003).

${ }^{28}$ H. T. C. Stoof and M. Bijlsma, Phys. Rev. E 47, 939 (1993).

${ }^{29}$ For a comprehensive review, see H. Shi and A. Griffin, Phys. Rep. 304, 1 (1998).

${ }^{30} \mathrm{M}$. Tinkham, Introduction to Superconductivy, 2nd ed. (McGrawHill, New York, 1996).

${ }^{31}$ J. P. Blaizot and G. Ripka, Quantum Theory of Finite Systems (MIT, Cambridge, MA, 1986).

${ }^{32} \mathrm{P}$. Nozières, in Bose-Einstein Condensation, edited by A. Griffin, D. W. Snoke, and S. Stringari (Cambridge University Press, Cambridge, 1996)

${ }^{33}$ G. Mahan, Many Particle Physics (Plenum, New York, 2000).

${ }^{34}$ E. Rezayi and N. Read, Phys. Rev. Lett. 72, 900 (1994).

${ }^{35}$ W. Kohn and D. Sherrington, Rev. Mod. Phys. 42, 1 (1970).

${ }^{36}$ S. M. Girvin, A. H. MacDonald, and P. M. Platzman, Phys. Rev. B 33, 2481 (1986).

${ }^{37}$ N. E. Bonesteel, I. A. McDonald, and C. Nayak, Phys. Rev. Lett. 77, 3009 (1996).

${ }^{38}$ V. I. Yukalov, Theor. Math. Phys. 37, 1093 (1978); Phys. A (Amsterdam) 100, 431 (1980).

${ }^{39}$ I. B. Spielman, L. A. Tracy, J. P. Eisenstein, L. N. Pfeiffer, and K. W. West, Phys. Rev. Lett. 94, 076803 (2005); N. Kumada, K. Muraki, K. Hashimoto, and Y. Hirayama, ibid. 94, 096802 (2005).

${ }^{40}$ P. Giudici, K. Muraki, N. Kumada, Y. Hirayama, and T. Fujisawa, Phys. Rev. Lett. 100, 106803 (2008). 
${ }^{41}$ P. Giudici, K. Muraki, N. Kumada, and T. Fujisawa, Phys. Rev. Lett. 104, 056802 (2010).

${ }^{42}$ R. L. Doretto and C. M. Smith, Phys. Rev. B 76, 195431 (2007).

${ }^{43}$ J. Sun, G. Murthy, H. A. Fertig, and N. Bray-Ali, Phys. Rev. B 81, 195314 (2010).

${ }^{44}$ T. Hyart and B. Rosenow, Phys. Rev. B 83, 155315 (2011).

${ }^{45}$ K. Moon, H. Mori, K. Yang, S. M. Girvin, A. H. MacDonald, L. Zheng, D. Yoshioka, and S.-C. Zhang, Phys. Rev. B 51, 5138 (1995).
${ }^{46}$ X. G. Wen and A. Zee, Phys. Rev. B 47, 2265 (1993); Z. F. Ezawa and A. Iwazaki, ibid. 48, 15189 (1993).

${ }^{47}$ A. A. Burkov and A. H. MacDonald, Phys. Rev. B 66, 115320 (2002); A. Burkov, J. Schliemann, A. H. MacDonald, and S. M. Girvin, Phys. E (Amsterdam) 12, 28 (2002).

${ }^{48}$ O. Tieleman, A. Lazarides, D. Makogon, and C. Morais Smith, Phys. Rev. B 80, 205315 (2009).

${ }^{49}$ T. T. Wu, Phys. Rev. 115, 1390 (1959).

${ }^{50}$ A. H. MacDonald, P. M. Platzman, and G. S. Boebinger, Phys. Rev. Lett. 65, 775 (1990). 